\title{
The Variety of Integrable Killing Tensors on the 3-Sphere
}

\author{
Konrad SCHÖBEL
}

Institut für Mathematik, Fakultät für Mathematik und Informatik, Friedrich-Schiller-Universität Jena, 07737 Jena, Germany

E-mail:konrad.schoebel@uni-jena.de

Received November 14, 2013, in final form July 15, 2014; Published online July 29, 2014 http://dx.doi.org/10.3842/SIGMA.2014.080

\begin{abstract}
Integrable Killing tensors are used to classify orthogonal coordinates in which the classical Hamilton-Jacobi equation can be solved by a separation of variables. We completely solve the Nijenhuis integrability conditions for Killing tensors on the sphere $S^{3}$ and give a set of isometry invariants for the integrability of a Killing tensor. We describe explicitly the space of solutions as well as its quotient under isometries as projective varieties and interpret their algebro-geometric properties in terms of Killing tensors. Furthermore, we identify all Stäckel systems in these varieties. This allows us to recover the known list of separation coordinates on $S^{3}$ in a simple and purely algebraic way. In particular, we prove that their moduli space is homeomorphic to the associahedron $K_{4}$.
\end{abstract}

Key words: separation of variables; Killing tensors; Stäckel systems; integrability; algebraic curvature tensors

2010 Mathematics Subject Classification: 53A60; 14H10; 14M12

\section{Contents}

1 Introduction $\quad \mathbf{2}$

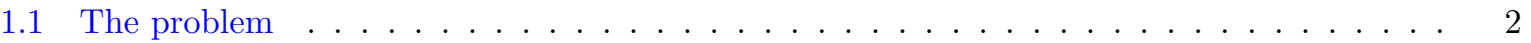

1.2 Prior results . . . . . . . . . . . . . . . . . . . . . . . . . 5

1.3 Method and results . . . . . . . . . . . . . . . . . . . . . . 6

1.4 Generalisations .............................. 9

1.5 Structure of the article . . . . . . . . . . . . . . . . . . . . . . 10

2 Properties of algebraic curvature tensors $\quad 10$

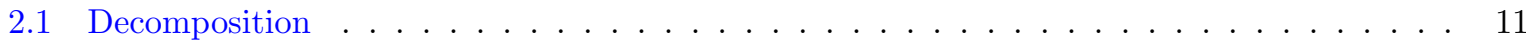

2.2 The action of the isometry group . . . . . . . . . . . . . . . . . . . . . . . . 12

2.3 Aligned algebraic curvature tensors . . . . . . . . . . . . . . . . . . . . . . . 14

2.4 Diagonal algebraic curvature tensors . . . . . . . . . . . . . . . . . . . . . . 15

2.5 The residual action of the isometry group . . . . . . . . . . . . . . . . . . 15

3 Solution of the algebraic integrability conditions 16

3.1 Reformulation of the first integrability condition . . . . . . . . . . . . . . . . 16

3.2 Integrability implies diagonalisability . . . . . . . . . . . . . . . . . . . . . . . . . 17

3.3 Solution of the second integrability condition . . . . . . . . . . . . . . . . . . . 19

4 The algebraic geometry of the KS-variety 21

5 Stäckel systems 25

5.1 Young tableaux . . . . . . . . . . . . . . . . . . . . . 26

5.2 Commuting Killing tensors . . . . . . . . . . . . . . . . . . . . 27 
6 Geometric constructions of integrable Killing tensors 32

6.1 Special Killing tensors . . . . . . . . . . . . . . . . . . . . . . . . . 32

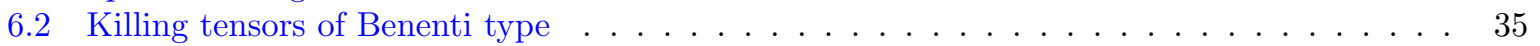

6.3 Benenti systems . . . . . . . . . . . . . . . . . . . . . . 36

6.4 Extension of integrable Killing tensors . . . . . . . . . . . . . . . . . . . . . . 39

7 Separation coordinates $\quad 40$

7.1 Eigenvalues of special conformal Killing tensors . . . . . . . . . . . . . . . . . . . . 40

7.2 Killing tensors and separation coordinates on $S^{2}$. . . . . . . . . . . . . . . . . . . 42

7.3 The classification . . . . . . . . . . . . . . . . . . . . . . . . . . 42

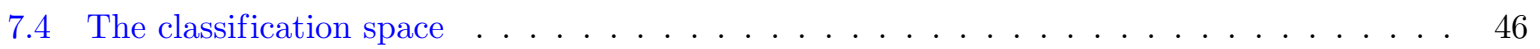

\section{Introduction}

\subsection{The problem}

Given a partial differential equation, it is a natural problem to seek for coordinate systems in which this equation can be solved by a separation of variables and to classify all such coordinate systems. It is not surprising, that for classical equations this problem has a very long history and is marked by the contributions of a number of prominent mathematicians, beginning with Gabriel Lamé in the first half of of the 19th century [29]. For example, consider the Hamilton-Jacobi equation

$$
\frac{1}{2} g^{i j} \frac{\partial S}{\partial x^{i}} \frac{\partial S}{\partial x^{j}}+V(x)=E
$$

on a Riemannian manifold. We say that it separates (additively) in a system of coordinates $x_{1}, \ldots, x_{n}$ if it admits a solution of the form

$$
S\left(x_{1}, \ldots, x_{n} ; \underline{c}\right)=\sum_{i=1}^{n} S_{i}\left(x_{i} ; \underline{c}\right), \quad \operatorname{det}\left(\frac{\partial^{2} S}{\partial x^{i} \partial c_{j}}\right) \neq 0,
$$

depending on $n$ constants $\underline{c}=\left(c_{1}, \ldots, c_{n}\right)$. If this is the case, the initial partial differential equation decouples into $n$ ordinary differential equations. We call the corresponding coordinate system separation coordinates.

It turns out that the Hamilton-Jacobi equation in the form (1.1) separates in a given orthogonal coordinate system if and only if it also separates for $V \equiv 0$ and if the potential $V$ satisfies a certain compatibility condition. In fact, the Hamilton-Jacobi equation with $V \equiv 0$ plays a key role for separation of variables in general. The reason is that for orthogonal coordinates the (additive) separation of this equation is a necessary condition for the (multiplicative) separation of other classical equations such as the Laplace equation, the Helmholtz equation or the Schrödinger equation. Sufficient conditions can be given in the form of compatibility conditions for the curvature tensor and for the potential.

The classification of orthogonal separation coordinates for the Hamilton-Jacobi equation thus leads to a variety of ordinary differential equations. These are well-known equations that define special functions appearing all over in mathematics and physics - such as Bessel functions, Legendre polynomials, spherical harmonics or Mathieu functions, to name just a few. In particular, these functions serve as bases for series expansions in the explicit solution of boundary value problems on domains bounded by coordinate hypersurfaces.

Separation coordinates are intimately related to the existence of rank two Killing tensors that satisfy a certain integrability condition. 
Definition 1.1. A symmetric tensor $K_{\alpha \beta}$ on a Riemannian manifold is a Killing tensor if and only if

$$
\nabla_{(\gamma} K_{\alpha \beta)}=0
$$

where $\nabla$ is the Levi-Civita connection of the metric $g$ and the round parenthesis denote complete symmetrisation of the enclosed indices. Depending on the context, we will consider a Killing tensor either as a symmetric bilinear form $K_{\alpha \beta}$ or alternatively as a symmetric endomorphism $K_{\beta}^{\alpha}=g^{\alpha \gamma} K_{\gamma \beta}$.

Definition 1.2. We say a diagonalisable endomorphism $K$ is integrable, if around any point in some open and dense set we find local coordinates such that the coordinate vectors are eigenvectors of $K$.

The above geometric definition of integrability can be cast into a system of partial differential equations, involving the Nijenhuis torsion of $K$ defined by

$$
N_{\beta \gamma}^{\alpha}=K_{\delta}^{\alpha} \nabla_{[\gamma} K_{\beta]}^{\delta}+\nabla_{\delta} K_{[\gamma}^{\alpha} K_{\beta]}^{\delta} .
$$

The following result was proven by Nijenhuis for endomorphisms with simple eigenvalues [37], but is true in general [41].

Proposition 1.3. A diagonalisable endomorphism field $K_{\beta}^{\alpha}$ on a Riemannian manifold is integrable if and only if it satisfies the Nijenhuis integrability conditions

$$
\begin{aligned}
& 0=N_{[\beta \gamma}^{\delta} g_{\alpha] \delta}, \\
& 0=N_{[\beta \gamma}^{\delta} K_{\alpha] \delta}, \\
& 0=N_{[\beta \gamma}^{\delta} K_{\alpha] \varepsilon} K_{\delta}^{\varepsilon},
\end{aligned}
$$

where the square brackets denote complete antisymmetrisation in the enclosed indices.

At the end of the 19th century, Paul Stäckel showed that any system of orthogonal separation coordinates gives rise to what we now call a Stäckel system. And in 1934, Luther P. Eisenhart proved the converse. This established a one-to-one correspondence between orthogonal separation coordinates and Stäckel systems in the following sense.

Definition 1.4. A Stäckel system on an $n$-dimensional Riemannian manifold is an $n$-dimensional space of integrable Killing tensors which mutually commute in the algebraic sense ${ }^{12}$.

Theorem $1.5([13,45])$. On a Riemannian manifold, there is a bijective correspondence between orthogonal separation coordinates and Stäckel systems.

Benenti later proved that a Stäckel system can be represented by a single integrable Killing tensor with simple eigenvalues. More precisely:

Theorem $1.6([3])$.

1. Every Stäckel system contains a Killing tensor with simple eigenvalues.

\footnotetext{
${ }^{1}$ Eisenhart's original definition of a Stäckel system was more restrictive, but is equivalent to the one given here [3].

${ }^{2}$ There is an alternative notion of "commuting" for Killing tensors, namely with respect to the Poisson bracket, which is different but closely related for a Stäckel system. Indeed, one can replace the condition that the Killing tensors in a Stäckel system are integrable by the condition that they mutually commute under the Poisson bracket [3]. In this article the term "commuting" will always be used in the algebraic sense.
} 
2. Conversely, every integrable Killing tensor with simple eigenvalues is contained in some Stäckel system.

3. A Stäckel system is uniquely determined by such a Killing tensor.

In particular, the separation coordinates can be recovered from a generic representative in a Stäckel system by solving the eigenproblem and integrating the distributions normal to the eigenvectors.

Although initially introduced to classify separation coordinates, Stäckel systems play a role far beyond separation of variables, as they constitute an important class of completely integrable dynamical systems. The construction and classification of Stäckel systems in constant curvature is, for instance, equivalent to the construction and classification of Gaudin magnets [21, 27, 28]. Stäckel systems also arise from Killing-Yano towers and in black hole integrable models [14]. Furthermore, there exists a natural quantisation of Killing tensors such that the quantum version of Stäckel systems are the maximal commutative subalgebras of second order symmetries of the Schrödinger operator [5].

The Nijenhuis integrability conditions (1.4) are homogeneous algebraic equations in $K$ and $\nabla K$, which are invariant under isometries. Separation of variables therefore naturally leads to the following problem.

Problem 1.7. Determine the projective variety $\mathcal{K}(M)$ of all integrable Killing tensors on a given Riemannian manifold $M$. That is, solve explicitly the Nijenhuis integrability conditions (1.4) for Killing tensors (1.2). Moreover, find natural isometry invariants characterising the integrability of a Killing tensor.

Of course, there are many examples of separation coordinates and in constant curvature we even have a complete classification. In principle this gives a description of the corresponding Stäckel systems and integrable Killing tensors, although in practice they are often obtained by intricate limiting processes. Nevertheless, this only yields a description of $\mathcal{K}(M)$ as a set, whereas in Problem 1.7 we seek to elucidate the natural algebraic geometric structure of this set. Note that in dimension two the Nijenhuis integrability conditions are void, so that $\mathcal{K}(M)$ is simply the projective space of Killing tensors. This means that Problem 1.7 is trivial in dimension two once the space of Killing tensors is known. However, to the best of our knowledge, already in dimensions three nothing is known about the set $\mathcal{K}(M)$ as a projective variety, apart from trivial cases, not even for Euclidean space $\mathbb{R}^{3}$ or the sphere $S^{3}$ - the two examples for which Eisenhart already derived the complete list of separation coordinates. Moreover, an explicit solution of the equations (1.4) has been considered intractable by various experts in this domain [15].

Although there exists no general construction for Stäckel systems, there is one which yields a large family of Stäckel systems. This family is interesting in itself, because it appears under different names in a number of different guises in the theory of integrable systems: as "Newtonian systems of quasi-Lagrangian type" [40], as "systems admitting special conformal Killing tensors" [8], as "cofactor systems" [31], as "bi-Hamiltonian structures" [6, 16], as "bi-quasiHamiltonian systems" [9, 10], as " $L$-systems" [4] or as "Benenti systems" [7]. We follow the latter nomenclature of Bolsinov and Matveev, because their description is best suited to our context. This brings us to the following question.

Question 1.8. Do Benenti systems parametrise the entire variety of integrable Killing tensors?

Moreover, by construction, the set of Benenti systems is not only invariant under isometries, but even under the projective group. So, if the answer to Question 1.8 is "no" and the isometry group is a proper subgroup of the projective group, the following question arises.

Question 1.9. Is the variety of integrable Killing tensors invariant under the projective group? 
Obviously, there are three groups acting on the variety $\mathcal{K}(M)$ of integrable Killing tensors:

1. $\mathbb{R}$, acting by addition of multiples of the metric $g$.

2. The isometry group $\operatorname{Isom}(M)$.

3. $\mathbb{R}^{*}$, acting by multiplication.

The actions 1 and 3 result from the fact that adding the identity to an endomorphism or multiplying it by a constant does not alter the eigenspaces and hence does not affect integrability. The actions 1 and 2 commute and the action 3 descends to the quotient. Hence the essential information about integrable Killing tensors is encoded in the projectivisation of the quotient

$$
\mathcal{K}(M) /(\operatorname{Isom}(M) \times \mathbb{R} g) .
$$

This leads to the following problem.

Problem 1.10. Determine explicitly the quotient (1.5), which describes integrable Killing tensors modulo obvious symmetries.

Recall that orthogonal separation coordinates are in bijective correspondence with Stäckel systems. Thus, in view of the classification problem for orthogonal separation coordinates, one is interested in linear subspaces of commuting integrable Killing tensors, rather than in the integrable Killing tensors themselves. This leads to the following problem.

Problem 1.11. Determine explicitly the space of all Stäckel systems on a given Riemannian manifold $M$ (modulo isometries). That is, find all maximal linear subspaces of commuting Killing tensors in $\mathcal{K}(M)$.

The separation coordinates can be recovered from an integrable Killing tensor with simple eigenvalues, because the coordinate hypersurfaces are orthogonal to the eigenvectors. This leads to the final step in the determination of separation coordinates via integrable Killing tensors and Stäckel systems.

Problem 1.12. Find a (canonical) representative in each Stäckel system for which it is possible to solve the eigenvalue problem and which has simple eigenvalues. Deduce the classification of orthogonal separation coordinates and give natural isometry invariants.

In this article we will completely solve Problems 1.7, 1.10, 1.11 and 1.12 for the threedimensional sphere $S^{3}$ equipped with the round metric and give answers to Questions 1.8 and 1.9. This is done without relying on any computer algebra. One of the reasons we choose $S^{3}$ is that it has the simplest isometry group among the 3-dimensional constant curvature manifolds. Our solution will reveal diverse facets of the problem of separation of variables, related to representation theory, algebraic geometry, geometric invariant theory, geodesic equivalence, Stasheff polytopes or moduli spaces of stable algebraic curves.

\subsection{Prior results}

In his fundamental paper Eisenhart also derived a list of orthogonal separation coordinates for $\mathbb{R}^{3}$ and $S^{3}$ [13]. Separation coordinates on $S^{3}$ were also classified in [38]. These results have been generalised by Kalnins and Miller, who gave an iterative diagrammatical procedure for the construction of orthogonal separation coordinates on spaces of constant curvature in any dimension $[17,22]$. Furthermore, Eisenhart computed the Stäckel systems on $\mathbb{R}^{3}$ in their respective separation coordinates. For $S^{3}$ the quantum point of view was adopted in [23]. 
In dimension two virtually everything is known. Koenigs classified all spaces with at least three independent Killing tensors. Apart from constant curvature spaces these are four socalled Darboux spaces and eleven multiparameter families of Koenigs spaces [26]. For Darboux spaces the separation coordinates have been classified in [19, 20] and for Koenigs spaces this is straightforward. Darboux spaces have analogs in higher dimensions [1], which can be obtained from constant curvature spaces via a Stäckel transform, and for the three dimensional version it is also straightforward to find all separation coordinates. Finally, superintegrability theory has led to the construction of non-conformally flat systems which are separable [18].

Despite these advances, the topology and the geometry of the space of integrable Killing tensors or its quotient by isometries has never been studied for any non-trivial case. Probably the only advance towards an algebraic geometric study of these spaces has been the construction of isometry invariants for Killing tensors which discriminate the different orthogonal separation coordinates in three dimensional Euclidean space [15]. This approach had not become viable until recently, because it relies on an extensive use of modern computer algebra. The algorithm, which depends on an a priori knowledge of the separation coordinates, involves the solution of a linear system of approximately 250,000 equations in 50,000 unknowns and the complexity of the algorithm renders it impractical in dimensions greater than three [11]. Nothing similar is known for the sphere or hyperbolic space. We are not aware of any applications of the resulting invariants to elucidate the structure of the resulting quotient.

\subsection{Method and results}

Our method is based on a purely algebraic description of the vector space of Killing tensors on constant curvature manifolds in combination with the corresponding algebraic equivalent of the Nijenhuis integrability conditions. We consider the standard 3-sphere, isometrically embedded as the unit sphere in a 4 -dimensional Euclidean vector space $(V, g)$ :

$$
S^{3}=\{x \in V: g(x, x)=1\} \subset V .
$$

The vector space of Killing tensors $K$ on $S^{3}$ is then naturally isomorphic to the vector space of algebraic curvature tensors on the ambient space $V$ [34]. These are (constant) tensors $R_{a_{1} b_{1} a_{2} b_{2}}$ on $V$ having the symmetries of a Riemannian curvature tensor. The isomorphism is explicitly given by

$$
K_{x}(v, w):=R_{a_{1} b_{1} a_{2} b_{2}} x^{a_{1}} x^{a_{2}} v^{b_{1}} w^{b_{2}}, \quad x \in S^{3}, \quad v, w \in T_{x} S^{3},
$$

and equivariant with respect to the natural actions of the isometry group $\mathrm{O}(V, g)$ on both spaces. Under this correspondence the Nijenhuis integrability conditions (1.4) for a Killing tensor are equivalent to the following algebraic integrability conditions for the associated algebraic curvature tensor [41]:

$$
\begin{aligned}
& \begin{array}{|l}
\frac{a_{2}}{b_{2}} \\
\frac{c_{2}}{d_{2}}
\end{array} g_{i j} R_{b_{1} a_{2} b_{2}}^{i} R_{d_{1} c_{2} d_{2}}^{j}=0,
\end{aligned}
$$

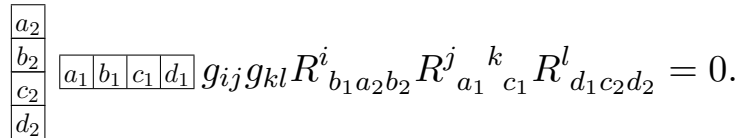

Here the Young symmetrisers on the left hand side denote complete antisymmetrisation in the indices $a_{2}, b_{2}, c_{2}, d_{2}$ respectively complete symmetrisation in the indices $a_{1}, b_{1}, c_{1}, d_{1}$. These homogeneous algebraic equations define the space of integrable Killing tensors as a projective variety. 
The key to the solution of the algebraic integrability conditions is a reinterpretion in terms of the decomposition of algebraic curvature tensors into irreducible representations of the isometry group - the selfdual and anti-selfdual Weyl part, the trace-free Ricci part and the scalar part. Under the action of the isometry group these components can be brought to a certain normal form, which simplifies considerably if the algebraic integrability conditions are imposed.

We regard an algebraic curvature tensor on $V$ as a symmetric endomorphism on $\Lambda^{2} V$. In this sense we will say that an algebraic curvature tensor $R$ is diagonal if $R_{i j k l}=0$ unless $\{i, j\}=\{k, l\}$. In dimension four a diagonal algebraic curvature tensor can be parametrised as

$$
R_{0 \alpha 0 \alpha}=w_{\alpha}+t_{\alpha}+\frac{s}{12}, \quad R_{\beta \gamma \beta \gamma}=w_{\alpha}-t_{\alpha}+\frac{s}{12},
$$

where $(\alpha, \beta, \gamma)$ denotes any cyclic permutation of $(1,2,3)$. In this parametrisation the $w_{\alpha}$ are the eigenvalues of the self-dual Weyl part, which turn out to be the same as those of the antiself-dual Weyl part, the $t_{\alpha}$ parametrise the eigenvalues of the trace free Ricci tensor and $s$ is the scalar curvature. In a first step we prove that diagonal algebraic curvature tensors define a (local) slice for the action of the isometry group on integrable Killing tensors.

Theorem 1.13 (integrability implies diagonalisability). Under the action of the isometry group any integrable Killing tensor on $S^{3}$ is equivalent to one with a diagonal algebraic curvature tensor.

The first algebraic integrability condition is identically satisfied on diagonal algebraic curvature tensors and the second reduces to a single algebraic equation on the diagonal entries. This yields a description of integrable Killing tensors with diagonal algebraic curvature tensor as a projective variety, equipped with the residual action of the isometry group. We call this variety the Killing-Stäckel variety (KS-variety).

Theorem 1.14 (integrability in the diagonal case). A Killing tensor on $S^{3}$ with diagonal algebraic curvature tensor (1.8) is integrable if and only if the associated matrix

$$
\left(\begin{array}{ccc}
\Delta_{1} & -t_{3} & t_{2} \\
t_{3} & \Delta_{2} & -t_{1} \\
-t_{2} & t_{1} & \Delta_{3}
\end{array}\right)
$$

has zero trace and zero determinant, where $\Delta_{1}:=w_{2}-w_{3}, \Delta_{2}:=w_{3}-w_{1}, \Delta_{3}:=w_{1}-w_{2}$. This defines a projective variety in $\mathbb{P}^{4}$ which carries a natural $S_{4}$-action, given by conjugating (1.9) with the symmetries of the regular octahedron in $\mathbb{R}^{3}$ with vertices $\pm e_{i}$ and adjacent faces oppositely oriented. Two diagonal algebraic curvature tensors are equivalent under the isometry group if and only if their associated matrices (1.9) are equivalent under this $S_{4}$-action.

Combining Theorems 1.13 and 1.14 yields a solution to Problem 1.10: The projectivisation of the quotient (1.5) is isomorphic to the quotient of the KS-variety by its natural $S_{4}$-action. Moreover, in the course of the proof of both theorems we establish isometry invariants for the integrability of a Killing tensor.

Proposition 1.15 (isometry invariants). Let $\mathbf{W}$ and $\mathbf{T}$ be the Weyl and Ricci part of the algebraic curvature tensor corresponding to a Killing tensor on $S^{3} \subset V$, regarded as endomorphisms on $\Lambda^{2} V$. Then this Killing tensor is integrable if and only if

1) $\operatorname{tr}[\mathbf{W}, \mathbf{T}]^{2}=0$,

2) $\operatorname{tr}\left(* \mathbf{W}^{2}\right)=\operatorname{tr}\left(* \mathbf{W}^{3}\right)=0$, where $*$ denotes the Hodge operator on $\Lambda^{2} V$ and

3) the endomorphisms $\mathbf{I}, \mathbf{W}$ and $\mathbf{W}^{2}-\mathbf{T}^{2}$ are linearly dependent. 
With this result the check whether a given Killing tensor on $S^{3}$ is integrable becomes algorithmically trivial.

Naïvely, one can find the Stäckel systems by solving the commutator equation $[K, \tilde{K}]=0$ for a fixed integrable Killing tensor $K$. But a far simpler way is to exploit the algebraic geometry of the KS-variety. Recall that Stäckel systems are linear spaces of dimension $n$ and always contain the metric. We show that the Killing tensors in a Stäckel system have simultaneously diagonalisable algebraic curvature tensors. Therefore Stäckel systems on $S^{3}$ correspond to certain projective lines in the KS-variety. In general, the projective lines in a projective variety constitute themselves a projective variety, called the Fano variety. That is, we are looking for a subvariety of the Fano variety of the KS-variety. By definition, the KS-variety is a linear section of the variety of $3 \times 3$ matrices with vanishing determinant, the so called full determinantal variety. Fortunately, the Fano variety of a full determinantal variety is well understood just in the case we need here.

So we only have to use the commutator equation to check which projective lines correspond to Stäckel systems, rather than to actually solve it. In order to do so, we derive an algebraic equivalent of the commutator equation in terms of the corresponding algebraic curvature tensors. This entails a detailed description of the algebraic geometry of the KS-variety and the Stäckel systems therein. In particular, we can relate the algebraic geometric properties of the KS-variety to the geometric properties of the corresponding integrable Killing tensors. For this, we remark that there is a natural way how to extend (integrable) Killing tensors from an embedded sphere $S^{m} \subset S^{n}$ to the ambient sphere $S^{n}$ for $m<n$.

Theorem 1.16. For $M=S^{3}$ the projectivisation of the quotient (1.5) is isomorphic to the quotient of the KS-variety by its natural action of $S_{4}$. Furthermore:

1. The singularities of the KS-variety correspond to extensions of the metric on $S^{2} \subset S^{3}$ respectively on $S^{1} \subset S^{3}$.

2. Stäckel systems determine projective lines in the KS-variety which consist of matrices (1.9) annihilating a given vector.

3. Projective planes in the KS-variety that consist of matrices (1.9) annihilating a fixed vector correspond to extensions of (integrable) Killing tensors from $S^{2}$ to $S^{3}$.

4. The projective plane of antisymmetric matrices in the KS-variety corresponds to the projective space of special Killing tensors. These are Killing tensors $K$ of the form

$$
K=L-(\operatorname{tr} L) g
$$

where $L$ is a special conformal Killing tensor ${ }^{3}$.

Based on the algebraic description of Stäckel systems we derive an algebraic description of Benenti systems as well and answer Question 1.8. The answer is "almost", in the following sense. On one hand, there are Stäckel systems on $S^{3}$, which are not Benenti systems. So the answer is clearly "no". But on the other hand these are exactly the extensions of Killing tensors from $S^{2}$ and for $S^{2}$ the answer is easily seen to be "yes".

From the algebraic description of Benenti systems we derive the following parametrisation of $\mathcal{K}\left(S^{3}\right)$, which is equivariant under the isometry group. This solves Problem 1.7.

Theorem 1.17 (equivariant parametrisation). The algebraic variety $\mathcal{K}\left(S^{3}\right)$ is the closure of the set of algebraic curvature tensors of the form

$$
\lambda_{2} h \otimes h+\lambda_{0} g \otimes g, \quad h \in \operatorname{Sym}^{2}(V), \quad \lambda_{0}, \lambda_{2} \in \mathbb{R} .
$$

\footnotetext{
${ }^{3}$ Cf. Definition 6.1.
} 
Here $₫$ denotes the Kulkarni-Nomizu product ${ }^{4}$ and $\frac{1}{2} g \otimes g$ is the algebraic curvature tensor of the metric. That is, $\mathcal{K}\left(S^{3}\right)$ is the closure of the projective cone from the metric over the image of the Kulkarni-Nomizu square $h \mapsto h \otimes h$, a map from symmetric tensors to algebraic curvature tensors. Remark that the apex lies itself in this image.

Since the set of algebraic curvature tensors of the form $h \otimes h$ is invariant under the projective group $\mathbb{P G L}(V)$ of $S^{3}$, the answer to Question 1.9 is "almost" as well. The reason is that although $\mathcal{K}\left(S^{3}\right)$ is not projectively invariant, this "invariant part" still contains the essential part of each Stäckel system unless it is an extension from $S^{2}$. But again, on $S^{2}$ the answer is easily seen to be "yes". Our answers to Questions 1.8 and 1.9 indicate that one should think of the space of integrable Killing tensors on the sphere as composed of strata, where the nongeneric strata come from lower dimensional spheres. Then each stratum is generated by Benenti systems and invariant under the projective group in the respective dimension.

As a simple consequence of Theorem 1.16 we get a canonical representative in each Stäckel system.

Corollary 1.18. Each Stäckel system on $S^{3}$ contains an essentially unique special Killing tensor.

If the eigenvalues of the corresponding special conformal Killing tensor are simple, they define the respective separation coordinates [8]. Moreover, every special conformal Killing tensor $L$ on $S^{n}$ is the restriction of a constant symmetric tensor $\hat{L}$ on the ambient space $V$ [32]. This is the reason why we can solve the eigenvalue problem for integrable Killing tensors explicitly. In this manner we get a purely algebraic classification of separation coordinates, which solves Problem 1.12. The details will be given later.

A solution to Problem 1.11 can be derived from the description of Stäckel systems in the KSvariety. This gives the classification space for separation coordinates on $S^{3}$ modulo isometries.

Theorem 1.19 (moduli space of separation coordinates). The space of Stäckel systems on $S^{3}$ modulo isometries is homeomorphic to the quotient of the blow-up of $\mathbb{P}^{2}$ in the four points $( \pm 1: \pm 1: \pm 1) \in \mathbb{P}^{2}$ under the natural action of the symmetry group $S_{4}$ of a tetrahedron in $\mathbb{R}^{3}$ inscribed in a cube with vertices $( \pm 1, \pm 1, \pm 1)$.

The fundamental domain of the $S_{4}$-action is a pentagon bounded by the non-generic separation coordinates. In fact, the multiplicities of the eigenvalues of the constant symmetric tensor $\hat{L}$ on $V$ identify this pentagon to the associahedron (or Stasheff polytope) $K_{4}$ [46]. These observations suggests a relation between separation coordinates on spheres and moduli spaces of stable curves of genus zero with marked points [12, 24, 25]. Indeed, our thorough analysis of the case $S^{3}$ presented here has led to the following generalisation of Theorem 1.19 [42].

Theorem 1.20. The Stäckel systems on $S^{n}$ with diagonal algebraic curvature tensor form a smooth projective variety isomorphic to the real Deligne-Mumford-Knudsen moduli space $\bar{M}_{0, n+2}(\mathbb{R})$ of stable genus zero curves with $n+2$ marked points.

\subsection{Generalisations}

Most of our results are stated for constant curvature manifolds of arbitrary dimension. What impedes a straightforward generalisation to higher dimensional spheres is that in the proof of diagonalisability we make essential use of the Hodge decomposition which only exists in dimension four. Theorem 1.20 together with a proof of the following conjecture would show, that the classification space for separation coordinates on $S^{n}$ is the associahedron $K_{n+1}$.

\footnotetext{
${ }^{4}$ Cf. Definition 6.6.
} 
Conjecture 1.21. Under the action of the isometry group any integrable Killing tensor on $S^{n}$ is equivalent to one with a diagonal algebraic curvature tensor.

In Lorentzian signature the Hodge star squares to minus one. A generalisation to hyperbolic space therefore naturally requires complexification. But over the complex numbers, all signatures are equivalent. This indicates that one should solve the algebraic integrability conditions over the complex numbers. The variety of complex solutions then contains not only the solution for hyperbolic space, but for all (non-flat) pseudo-Riemannian constant curvature spaces as real subvarieties, singled out by appropriate choices of a real structure. In dimension three there is an elegant way to do this using spinors and the Petrov classification of curvature tensors [35]. The main complication comes from the fact that diagonalisability does not hold anymore in the complexified case.

A challenge remains without doubt a non-trivial algebraic geometric classification of integrable Killing tensors and orthogonal separation coordinates on some non-constant curvature manifold. This is out of scope of common, purely geometric techniques. Note that the Nijenhuis integrability conditions (1.4) can always be rewritten as homogeneous algebraic equations of degree two, three and four on the space of Killing tensors, invariant under the isometry group action on Killing tensors. This endows the set of integrable Killing tensors on an arbitrary Riemannian manifold with the structure of a projective variety, equipped with an action of the isometry group. Our approach can therefore be extended to non-constant curvature as well. Natural candidates are semi-simple Lie groups, homogeneous spaces or Einstein manifolds. Notice that $S^{3}$ falls in either of these categories. Taking our solution for $S^{3}$ as a roadmap, we propose the following three-stage procedure:

1. Determine the space of Killing tensors and identify it explicitly as a representation of the isometry group (as in [34] for constant curvature manifolds).

2. Translate the Nijenhuis integrability conditions for Killing tensors into purely algebraic integrability conditions (as in [41] for constant curvature manifolds).

3. Solve the algebraic integrability conditions, study the algebraic geometric properties of the resulting variety and derive the complete classification of orthogonal separation coordinates (as in this article for $S^{3}$ ).

We consider the present work as a proof of concept that this algebraic geometric approach to the problem of separation of variables is viable.

\section{$1.5 \quad$ Structure of the article}

The article is organised as follows: In Section 2 we examine some properties of algebraic curvature tensors that will be used to solve the algebraic integrability conditions in Section 3 . We then describe the algebraic geometry of this solution in Section 4 and identify the Stäckel systems therein in Section 5. In Section 6 we give various geometric constructions for integrable Killing tensors and interpret them algebraically. In the last section we demonstrate how the classification of separation coordinates can be recovered within our framework.

\section{Properties of algebraic curvature tensors}

Algebraic curvature tensors on a vector space $V$ are four-fold covariant tensors $R_{a_{1} b_{1} a_{2} b_{2}}$ having the same algebraic symmetries as a Riemannian curvature tensor:

$$
\begin{array}{ll}
\text { antisymmetry: } & R_{b_{1} a_{1} a_{2} b_{2}}=-R_{a_{1} b_{1} a_{2} b_{2}}=R_{a_{1} b_{1} b_{2} a_{2}}, \\
\text { pair symmetry: } & R_{a_{2} b_{2} a_{1} b_{1}}=R_{a_{1} b_{1} a_{2} b_{2}}, \\
\text { Bianchi identity: } & R_{a_{1} b_{1} a_{2} b_{2}}+R_{a_{1} a_{2} b_{2} b_{1}}+R_{a_{1} b_{2} b_{1} a_{2}}=0 .
\end{array}
$$


Given a scalar product $g$ on $V$, we can raise and lower indices. The symmetries (2.1a) and (2.1b) then allow us to regard an algebraic curvature tensor $R_{a_{1} a_{2} b_{1} b_{2}}$ on $V$ as a symmetric endomorphism $R_{a_{2} b_{2}}^{a_{1} b_{1}}$ on the space $\Lambda^{2} V$ of 2 -forms on $V$. Since we will frequently change between both interpretations, we denote endomorphisms by the same letter in boldface.

\subsection{Decomposition}

In the special case where $\operatorname{dim} V=4$, the Hodge star operator "*” defines a decomposition

$$
\Lambda^{2} V=\Lambda_{+}^{2} V \oplus \Lambda_{-}^{2} V
$$

of $\Lambda^{2} V$ into its \pm 1 eigenspaces. We can therefore write an algebraic curvature tensor $R$ and the Hodge star as block matrices

$$
\mathbf{R}=\left(\begin{array}{c|c}
W_{+} & T_{\mp} \\
\hline T_{ \pm} & W_{-}
\end{array}\right)+\frac{s}{12}\left(\begin{array}{c|c}
I_{+} & 0 \\
\hline 0 & I_{-}
\end{array}\right), \quad *=\left(\begin{array}{c|c}
+I_{+} & 0 \\
\hline 0 & -I_{-}
\end{array}\right)
$$

with the $3 \times 3$-blocks satisfying

$$
\begin{aligned}
& W_{+}^{t}=W_{+}, \quad W_{-}^{t}=W_{-}, \quad T_{\mp}=T_{ \pm}^{t}, \\
& \operatorname{tr} W_{+}+\operatorname{tr} W_{-}=0, \\
& \operatorname{tr} W_{+}-\operatorname{tr} W_{-}=0 .
\end{aligned}
$$

Here $I_{+}$and $I_{-}$denote the identity on $\Lambda_{+}^{2} V$ respectively $\Lambda_{-}^{2} V$. The conditions $(2.2 \mathrm{~b})$ assure symmetry, condition (2.2c) says that $s=2 \operatorname{tr} \mathbf{R}$ and condition (2.2d) is a reformulation of the Bianchi identity (2.1c). Indeed, the Bianchi identity is equivalent to the vanishing of the antisymmetrisation of $R_{a_{1} b_{1} a_{2} b_{2}}$ in all four indices. In dimension four this can be written as $\varepsilon^{a_{2} b_{2} a_{1} b_{1}} R_{a_{1} b_{1} a_{2} b_{2}}=0$ or

$$
\varepsilon^{a_{2} b_{2}}{ }_{a_{1} b_{1}} \mathbf{R}^{a_{1} b_{1}}{ }_{a_{2} b_{2}}=0
$$

where $\varepsilon^{a_{2} b_{2} a_{1} b_{1}}$ is the totally antisymmetric tensor. Notice that $\varepsilon^{a_{2} b_{2}}{ }_{a_{1} b_{1}}$ is nothing else than the Hodge star operator and hence

$$
\operatorname{tr}(* \mathbf{R})=0
$$

where tr $: \operatorname{End}\left(\Lambda^{2} V\right) \rightarrow \mathbb{R}$ is the usual trace. Now (2.2d) is (2.3) applied to $\mathbf{R}$ in (2.2).

The space of algebraic curvature tensors is an irreducible GL( $V)$-representation and (2.2) gives a decomposition of this representation into irreducible representations of the subgroup $\mathrm{SO}(V, g)$ when $\operatorname{dim} V=4$. As the notation already suggests, we can relate these components to the familiar Ricci decomposition

$$
\mathbf{R}=\mathbf{W}+\mathbf{T}+\mathbf{S}
$$

of an algebraic curvature tensor $R$ into

- a scalar part

$$
S_{a_{1} b_{1} a_{2} b_{2}}:=\frac{s}{12}\left(g_{a_{1} a_{2}} g_{b_{1} b_{2}}-g_{a_{1} b_{2}} g_{b_{1} a_{2}}\right)
$$

given by the scalar curvature

$$
s=g^{a_{1} a_{2}} g^{b_{1} b_{2}} R_{a_{1} b_{1} a_{2} b_{2}},
$$


- a trace free Ricci part

$$
T_{a_{1} b_{1} a_{2} b_{2}}=\frac{1}{2}\left(T_{a_{1} a_{2}} g_{b_{1} b_{2}}-T_{a_{1} b_{2}} g_{b_{1} a_{2}}-T_{b_{1} a_{2}} g_{a_{1} b_{2}}+T_{b_{1} b_{2}} g_{a_{1} a_{2}}\right)
$$

given by the trace free Ricci tensor

$$
T_{a_{1} a_{2}}=g^{b_{1} b_{2}} R_{a_{1} b_{1} a_{2} b_{2}}-\frac{s}{4} g_{a_{1} a_{2}},
$$

- and a Weyl part, given by the totally trace free Weyl tensor $W:=R-T-S$.

It is not difficult to check that $[*, \mathbf{T}]=0$ and hence

$$
\mathbf{W}=\left(\begin{array}{c|c}
W_{+} & 0 \\
\hline 0 & W_{-}
\end{array}\right), \quad \mathbf{T}=\left(\begin{array}{c|c}
0 & T_{\mp} \\
\hline T_{ \pm} & 0
\end{array}\right), \quad \mathbf{S}=\frac{s}{12}\left(\begin{array}{c|c}
I_{+} & 0 \\
\hline 0 & I_{-}
\end{array}\right) .
$$

We see that $W_{+}$and $W_{-}$are the self-dual and anti-self-dual part of the Weyl tensor. Implicitly, the above interpretation also provides an isomorphism

$$
\begin{aligned}
\operatorname{Sym}_{0}(V) & \cong \operatorname{Hom}\left(\Lambda_{+}^{2} V, \Lambda_{-}^{2} V\right), \\
T & \mapsto T_{ \pm},
\end{aligned}
$$

between trace-free Ricci tensors and homomorphisms from self-dual to anti-self-dual 2-forms.

\subsection{The action of the isometry group}

The Killing tensor equation (1.2) as well as the integrability conditions (1.4) are invariant under the action of the isometry group. In other words, the variety of integrable Killing tensors is invariant under isometries. This will allow us later to put the algebraic curvature tensor of an integrable Killing tensor on $S^{3}$ to a certain normal form and to solve the algebraic integrability conditions for this particular form. Recall that the isometry group of $S^{n} \subset V$ is $\mathrm{O}(V)$ and that $\mathrm{SO}(V)$ is the subgroup of orientation preserving isometries.

We first examine the induced action of $\mathrm{SO}(V)$ on algebraic curvature tensors of the form (2.2). The standard action of $\mathrm{SO}(V)$ on $V$ induces a natural action on $\Lambda^{2} V$ which is the adjoint action of $\mathrm{SO}(V)$ on its Lie algebra under the isomorphism

$$
\mathfrak{s o}(V) \cong \Lambda^{2} V .
$$

Now consider the following commutative diagram of Lie group morphisms for $\operatorname{dim} V=4$ :

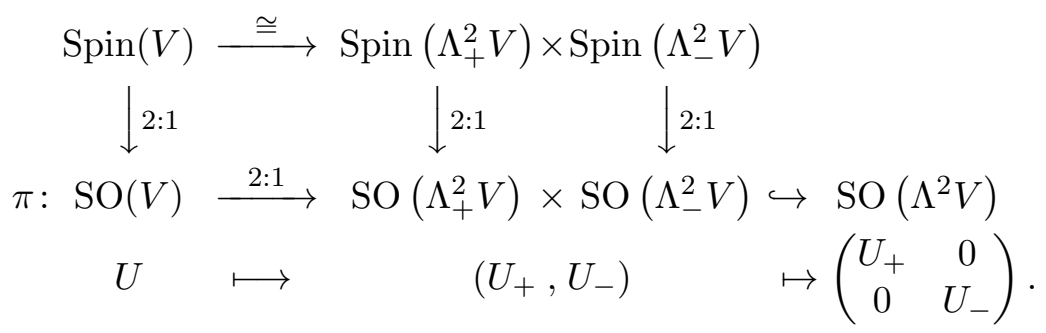

Here the double covering $\pi$ in the second row is induced from the exceptional isomorphism in the first row via the universal covering maps (vertical). Under the induced isomorphism $\pi_{*}$ of Lie algebras,

$$
\mathfrak{s o}(V) \cong \mathfrak{s o}\left(\Lambda_{+}^{2} V\right) \oplus \mathfrak{s o}\left(\Lambda_{-}^{2} V\right)
$$


the adjoint action of an element $U \in \mathrm{SO}(V)$ corresponds to the adjoint actions of $U_{+} \in \mathrm{SO}\left(\Lambda_{+}^{2} V\right)$ and $U_{-} \in \mathrm{SO}\left(\Lambda_{-}^{2} V\right)$. Since $\Lambda_{+}^{2} V$ has dimension three, the Hodge star operator gives an isomorphism

$$
\mathfrak{s o}\left(\Lambda_{+}^{2} V\right) \cong \Lambda^{2} \Lambda_{+}^{2} V \cong \Lambda_{+}^{2} V
$$

under which the adjoint action of $U_{+}$corresponds to the standard action on $\Lambda_{+}^{2} V$ and similarly for $U_{-}$. The Hodge decomposition completes the above isomorphisms to a commutative diagram

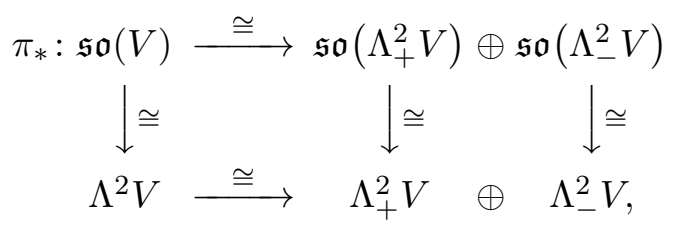

which shows that the natural action of $U \in \mathrm{SO}(V)$ on $\Lambda^{2} V$ is given by

$$
\left(\begin{array}{cc}
U_{+} & 0 \\
0 & U_{-}
\end{array}\right) \in \pi(\mathrm{SO}(V)) \subset \mathrm{SO}\left(\Lambda^{2} V\right)
$$

Hence the natural action of $U$ on $\operatorname{End}\left(\Lambda^{2} V\right)$ is given by conjugation with this matrix. Restricting to algebraic curvature tensors we get:

Proposition 2.1 (action of the isometry group). An element $U \in \mathrm{SO}(V)$ acts on algebraic curvature tensors in the form (2.2) by conjugation with (2.6), i.e. via

$$
W_{+} \mapsto U_{+}^{t} W_{+} U_{+}, \quad W_{-} \mapsto U_{-}^{t} W_{-} U_{-}, \quad T_{ \pm} \mapsto U_{-}^{t} T_{ \pm} U_{+}
$$

where $\pi(U)=\left(U_{+}, U_{-}\right)$is the image of $U$ under the double covering

$$
\pi: \quad \mathrm{SO}(V) \rightarrow \mathrm{SO}\left(\Lambda_{+}^{2} V\right) \times \mathrm{SO}\left(\Lambda_{-}^{2} V\right) .
$$

We can describe the twofold cover $\pi$ explicitly in terms of orthonormal bases on the spaces $V$, $\Lambda_{+}^{2} V$ and $\Lambda_{-}^{2} V$. It maps an orthonormal basis $\left(e_{0}, e_{1}, e_{2}, e_{3}\right)$ of $V$ to the orthonormal bases $\left(\eta_{+1}, \eta_{+2}, \eta_{+3}\right)$ of $\Lambda_{+}^{2} V$ and $\left(\eta_{-1}, \eta_{-2}, \eta_{-3}\right)$ of $\Lambda_{-}^{2} V$, defined by

$$
\eta_{ \pm \alpha}:=\frac{1}{\sqrt{2}}\left(e_{0} \wedge e_{\alpha} \pm e_{\beta} \wedge e_{\gamma}\right)
$$

for each cyclic permutation $(\alpha, \beta, \gamma)$ of $(1,2,3)$. From this description also follows that ker $\pi=$ $\{ \pm I\}$.

The action of $\mathrm{O}(V)$ on algebraic curvature tensors is now determined by the action of some orientation reversing element in $\mathrm{O}(V)$, say the one given by reversing the sign of $e_{0}$ and preserving $e_{1}, e_{2}$ and $e_{3}$. This element maps $\eta_{ \pm \alpha}$ to $-\eta_{\mp \alpha}$. Hence its action on algebraic curvature tensors in the form (2.2) is given by conjugation with

$$
\left(\begin{array}{cc}
0 & -I \\
-I & 0
\end{array}\right)
$$

respectively by mapping

$$
W_{+} \mapsto W_{-}, \quad T_{ \pm} \mapsto T_{\mp} .
$$




\subsection{Aligned algebraic curvature tensors}

$W_{+}$and $W_{-}$are symmetric and hence simultaneously diagonalisable under the action (2.7) of $\mathrm{SO}(V)$. On the other hand, the singular value decomposition shows that $T_{ \pm}$is also diagonalisable under this action, although in general not simultaneously with $W_{+}$and $W_{-}$. The following lemma gives a criterion when this is the case.

Lemma 2.2. Let $W_{+}$and $W_{-}$be symmetric endomorphisms on two arbitrary Euclidean vector spaces $\Lambda_{+}$respectively $\Lambda_{-}$and suppose the linear map $T_{ \pm}: \Lambda_{+} \rightarrow \Lambda_{-}$satisf ies

$$
T_{ \pm} W_{+}=W_{-} T_{ \pm}
$$

Then there exist orthonormal bases for $\Lambda_{+}$and $\Lambda_{-}$such that $W_{+}, W_{-}$and $T_{ \pm}$are simultaneously diagonal with respect to these bases.

Proof. It suffices to show that we can chose diagonal bases for $W_{+}$and $W_{-}$such that the matrix of $T_{ \pm}$has at most one non-zero element in each row and in each column, for the desired result can then be obtained by an appropriate permutation of the basis elements. The above condition implies that $T_{ \pm}$maps eigenspaces of $W_{+}$to eigenspaces of $W_{-}$with the same eigenvalue. Without loss of generality we can thus assume that $\Lambda_{+}$and $\Lambda_{-}$are eigenspaces of $W_{+}$respectively $W_{-}$with the same eigenvalue. But then $W_{+}$and $W_{-}$are each proportional to the identity and therefore diagonal in any basis. In this case the lemma follows from the singular value decomposition of $T_{ \pm}$.

Lemma 2.3. The following conditions are equivalent for an algebraic curvature tensor (2.2):

1) $T_{ \pm} W_{+}=W_{-} T_{ \pm}$,

2) $[\mathbf{W}, \mathbf{T}]=0$,

3) $\mathbf{W}$ and $\mathbf{T}$ are simultaneously diagonalisable under $\mathrm{SO}\left(\Lambda^{2} V\right)$.

Proof. The equivalence of 1 and 2 follows from (2.4). For the equivalence of 2 and 3 it suffices to note that $\mathbf{W}$ and $\mathbf{T}$ are both symmetric and hence diagonalisable.

The following terminology is borrowed from general relativity.

Definition 2.4. We say that an algebraic curvature tensor is aligned, if it satisfies one of the equivalent conditions in Lemma 2.3.

By Lemma 2.3, for any aligned algebraic curvature tensor we can find an orthonormal basis of $V$ such that

$$
W_{+}=\left(\begin{array}{ccc}
w_{+1} & 0 & 0 \\
0 & w_{+2} & 0 \\
0 & 0 & w_{+3}
\end{array}\right), \quad W_{-}=\left(\begin{array}{ccc}
w_{-1} & 0 & 0 \\
0 & w_{-2} & 0 \\
0 & 0 & w_{-3}
\end{array}\right), \quad T_{ \pm}=\left(\begin{array}{ccc}
t_{1} & 0 & 0 \\
0 & t_{2} & 0 \\
0 & 0 & t_{3}
\end{array}\right)
$$

with

$$
w_{+1}+w_{+2}+w_{+3}=0, \quad w_{-1}+w_{-2}+w_{-3}=0 .
$$

To simplify notation we agree that henceforth the indices $(\alpha, \beta, \gamma)$ will stand for an arbitrary cyclic permutation of $(1,2,3)$. Changing the basis in $\Lambda^{2} V$ from (2.8) to $e_{i} \wedge e_{j}, 0 \leqslant i<j \leqslant 4$, we obtain the independent components of an aligned algebraic curvature tensor:

$$
\begin{aligned}
& R_{0 \alpha 0 \alpha}=\frac{w_{+\alpha}+w_{-\alpha}}{2}+t_{\alpha}+\frac{s}{12}, \quad R_{\beta \gamma \beta \gamma}=\frac{w_{+\alpha}+w_{-\alpha}}{2}-t_{\alpha}+\frac{s}{12}, \\
& R_{0 \alpha \beta \gamma}=\frac{w_{+\alpha}-w_{-\alpha}}{2} .
\end{aligned}
$$


The trace free Ricci tensor of this aligned algebraic curvature tensor is diagonal and given by

$$
T_{00}=t_{\alpha}+t_{\beta}+t_{\gamma}, \quad T_{\alpha \alpha}=t_{\alpha}-t_{\beta}-t_{\gamma}, \quad t_{\alpha}=\frac{T_{00}+T_{\alpha \alpha}}{2} .
$$

This is nothing else than the restriction of the isomorphism (2.5) to diagonal tensors.

\subsection{Diagonal algebraic curvature tensors}

Definition 2.5. We call an algebraic curvature tensor on $V$ diagonal in a basis $e_{i}$ if it is diagonal as an element of $\operatorname{End}\left(\Lambda^{2} V\right)$ with respect to the associated basis $e_{i} \wedge e_{j}, i<j$. We call it diagonalisable if it is diagonalisable under the adjoint action of $\mathrm{SO}(V)$ on $\Lambda^{2} V$, i.e. under the subgroup $\pi(\mathrm{SO}(V)) \subset \mathrm{SO}\left(\Lambda^{2} V\right)$.

Of course, being a symmetric endomorphism on $\Lambda^{2} V$, an algebraic curvature tensor is always diagonalisable under the full group $\mathrm{SO}\left(\Lambda^{2} V\right)$.

Proposition 2.6 (diagonalisability criterion). An algebraic curvature tensor is diagonalisable if and only if it is aligned and $W_{+}$has the same characteristic polynomial as $W_{-}$.

Proof. Consider an aligned algebraic curvature tensor and suppose that $W_{+}$has the same characteristic polynomial as $W_{-}$. Then we can find a transformation (2.7) such that, as $3 \times 3$ matrices, $W_{+}=W_{-}$. The condition $W_{-} T_{\mp}=T_{ \pm} W_{+}$then implies that $T_{ \pm}$can be simultaneously diagonalised with $W_{+}=W_{-}$. This means we can assume without loss of generality that

$$
w_{+\alpha}=w_{-\alpha}=: w_{\alpha}
$$

for $\alpha=1,2,3$ in $(2.10)$, so that (2.11) reads

$$
R_{0 \alpha 0 \alpha}=w_{\alpha}+t_{\alpha}+\frac{s}{12}, \quad R_{0 \alpha \beta \gamma}=0, \quad R_{\beta \gamma \beta \gamma}=w_{\alpha}-t_{\alpha}+\frac{s}{12}
$$

with $w_{\alpha}$ subject to

$$
w_{1}+w_{2}+w_{3}=0 .
$$

Obviously this algebraic curvature tensor is diagonal. On the other hand, any diagonal algebraic curvature tensor can be written in the form (2.13), because

$$
\begin{aligned}
& s=2\left(R_{0101}+R_{2323}+R_{0202}+R_{3131}+R_{0303}+R_{1212}\right), \\
& t_{\alpha}=\frac{R_{0 \alpha 0 \alpha}-R_{\beta \gamma \beta \gamma}}{2}, \quad w_{\alpha}=\frac{R_{0 \alpha 0 \alpha}+R_{\beta \gamma \beta \gamma}}{2}-\frac{s}{12} .
\end{aligned}
$$

\subsection{The residual action of the isometry group}

For later use we need the stabiliser of the space of diagonal algebraic curvature tensors under the action of the isometry group. Such tensors are of the form (2.10) with $w_{-\alpha}=w_{+\alpha}$ and are invariant under the transformation (2.9). Hence it is sufficient to consider only the subgroup $\mathrm{SO}(V)$ of orientation preserving isometries.

Let $U \in \mathrm{SO}(V)$ with $\pi(U)=\left(U_{+}, U_{-}\right)$be an element preserving the space of diagonal algebraic curvature tensors under the action (2.7). Then $U_{+} \in \mathrm{SO}\left(\Lambda_{+}^{2} V\right)$ and $U_{-} \in \mathrm{SO}\left(\Lambda_{-}^{2} V\right)$ preserve the space of diagonal matrices on $\Lambda_{+}^{2} V$ respectively $\Lambda_{-}^{2} V$. In the orthogonal group, the stabiliser of the space of diagonal matrices under conjugation is the subgroup of signed permutation matrices. They act by permuting the diagonal elements in disregard of the signs. In particular, in $\mathrm{SO}(3)$ the stabiliser subgroup of the space of diagonal matrices on $\mathbb{R}^{3}$ is isomorphic 
to $S_{4}$. Under the isomorphism $S_{4} \cong S_{3} \ltimes V_{4}$, the permutation group $S_{3}$ is the subgroup of (unsigned) permutation matrices and acts by permuting the diagonal elements, whereas the Klein four group $V_{4}$ is the subgroup of diagonal matrices in $\mathrm{SO}(3)$ and acts trivially.

Using the above, it is not difficult to see that the stabiliser of the space of diagonal algebraic curvature tensors under the action (2.7) is isomorphic to $S_{4} \times \operatorname{ker} \pi$, with $\operatorname{ker} \pi=\{ \pm I\}$ acting trivially. Under the isomorphism $S_{4} \cong S_{3} \ltimes V_{4}$, the factor $S_{3}$ acts by simultaneous permutations of $\left(w_{1}, w_{2}, w_{3}\right)$ and $\left(t_{1}, t_{2}, t_{3}\right)$ and the factor $V_{4}$ by simultaneous flips of two signs in $\left(t_{1}, t_{2}, t_{3}\right)$.

\section{Solution of the algebraic integrability conditions}

This section is dedicated to proving Theorems 1.13 and 1.14 .

\subsection{Reformulation of the first integrability condition}

In the same way as for the Bianchi identity we can reformulate the first integrability condition (1.7a) as

$$
\mathbf{R}_{a_{2} b_{2}}^{i b_{1}} \varepsilon_{c_{2} d_{2}}^{a_{2} b_{2}} \mathbf{R}^{c_{2} d_{2}}{ }_{i d_{1}}=0
$$

or

$$
r(\mathbf{R} * \mathbf{R})=0,
$$

where

$$
\begin{aligned}
r: \quad \operatorname{End}\left(\Lambda^{2} V\right) & \longrightarrow \operatorname{End}(V), \\
\mathbf{E}^{i j}{ }_{k l} & \mapsto \mathbf{E}^{i j}{ }_{k j}
\end{aligned}
$$

denotes the Ricci contraction. Notice that $\mathbf{E}:=\mathbf{R} * \mathbf{R}$ is symmetric, but does in general not satisfy the Bianchi identity. A proof of the following lemma can be found in [43].

Lemma 3.1. The kernel of the Ricci contraction (3.2) is composed of endomorphisms $\mathbf{E}$ satisfying

$$
* \mathbf{E} *=\mathbf{E}^{t}, \quad \operatorname{tr} \mathbf{E}=0 .
$$

Applying this to the symmetric endomorphism

$$
\mathbf{E}:=\mathbf{R} * \mathbf{R}=\left(\begin{array}{c|c}
\left(W_{+}+\frac{s}{12} I_{+}\right)^{2}-T_{\mp} T_{ \pm} & \begin{array}{c}
\left(W_{+}+\frac{s}{12} I_{+}\right) T_{\mp} \\
-T_{\mp}\left(W_{-}+\frac{s}{12} I_{-}\right)
\end{array} \\
\hline T_{ \pm}\left(W_{+}+\frac{s}{12} I_{+}\right) & T_{ \pm} T_{\mp}-\left(W_{-}+\frac{s}{12} I_{-}\right)^{2} \\
-\left(W_{-}+\frac{s}{12} I_{-}\right) T_{ \pm} &
\end{array},\right.
$$

in (3.1) and using (2.2c) we get

$$
\begin{aligned}
& W_{+} T_{\mp}=T_{\mp} W_{-}, \\
& \operatorname{tr} W_{+}^{2}=\operatorname{tr} W_{-}^{2} .
\end{aligned}
$$

This shows:

Proposition 3.2. The first algebraic integrability condition for an algebraic curvature tensor $\mathbf{R}=\mathbf{W}+\mathbf{T}$ with Weyl part $\mathbf{W}$ and (not necessarily trace free) Ricci part $\mathbf{T}$ is equivalent to

$$
[\mathbf{W}, \mathbf{T}]=0, \quad \operatorname{tr}\left(* \mathbf{W}^{2}\right)=0 .
$$

In particular, an algebraic curvature tensor satisfying the first algebraic integrability condition is aligned. 
As a consequence of Proposition 2.6 we get:

Corollary 3.3. A diagonalisable algebraic curvature tensor satisfies the first algebraic integrability condition.

\subsection{Integrability implies diagonalisability}

The aim of this subsection is to prove Theorem 1.13. In view of Propositions 2.6 and 3.2 it is sufficient to show that if an algebraic curvature tensor satisfies the algebraic integrability conditions, then $W_{+}$and $W_{-}$have the same characteristic polynomial. We will first prove that the first algebraic integrability condition implies that $W_{+}$has the same characteristic polynomial either as $+W_{-}$or as $-W_{-}$. We then prove that the latter contradicts the second algebraic integrability condition.

We saw that an algebraic curvature tensor which satisfies the first algebraic integrability condition is aligned and thus of the form (2.10) in a suitable orthogonal basis. The first integrability condition in the form (3.3) then translates to

$$
w_{+1}^{2}+w_{+2}^{2}+w_{+3}^{2}=r^{2}, \quad w_{-1}^{2}+w_{-2}^{2}+w_{-3}^{2}=r^{2}
$$

for some $r \geqslant 0$ and

$$
w_{+\alpha} t_{\alpha}=t_{\alpha} w_{-\alpha}
$$

If we regard $\left(w_{+1}, w_{+2}, w_{+3}\right)$ and $\left(w_{-1}, w_{-2}, w_{-3}\right)$ as vectors in $\mathbb{R}^{3}$, then each equation in $(2.10 \mathrm{~b})$ describes the plane through the origin with normal $(1,1,1)$ and each equation in $(3.4 \mathrm{a})$ the sphere of radius $r$ centered at the origin. Hence the solutions to both equations lie on a circle and can be parametrised in polar coordinates by a radius $r \geqslant 0$ and angles $\varphi_{+}, \varphi_{-}$as

$$
w_{+\alpha}=r \cos \left(\varphi_{+}+\alpha \frac{2 \pi}{3}\right), \quad w_{-\alpha}=r \cos \left(\varphi_{-}+\alpha \frac{2 \pi}{3}\right) .
$$

Assume now that $W_{+}$and $W_{-}$have different eigenvalues. Then we have $T_{ \pm}=0$. Indeed, if $t_{\alpha} \neq 0$ for some $\alpha$, then (3.4b) shows that $w_{+\alpha}=w_{-\alpha}$ for this index $\alpha$. But then (3.5) implies that $W_{+}$and $W_{-}$have the same eigenvalues, which contradicts our assumption. Hence $T_{ \pm}=0$.

Without loss of generality we will set $s=0$ in what follows, because integrability is independent of the scalar curvature $s$. This follows from the fact that the Killing tensor corresponding to the scalar component in the Ricci decomposition is proportional to the metric and thus to the identity when regarded as an endomorphism. But adding a multiple of the identity only alters the eigenvalues, not the eigendirections, and therefore has no effect on integrability.

After these considerations, the independent components (2.11) of our algebraic curvature tensor are

$$
R_{0 \alpha 0 \alpha}=\frac{w_{+\alpha}+w_{-\alpha}}{2}=R_{\beta \gamma \beta \gamma}, \quad R_{0 \alpha \beta \gamma}=\frac{w_{+\alpha}-w_{-\alpha}}{2} .
$$

Choosing $a_{1}=b_{1}=c_{1}=d_{1}=0$ in the second integrability condition (1.7b) for this algebraic curvature tensor yields

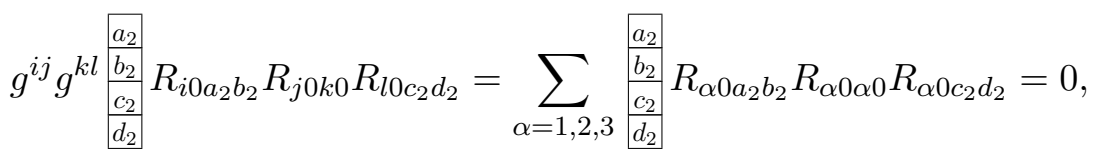

since only terms with $i=j=k=l \neq 0$ contribute to the contraction. For $\left(a_{2}, b_{2}, c_{2}, d_{2}\right)=$ $(0,1,2,3)$ this is gives

$$
\sum R_{\alpha 00 \alpha} R_{\alpha 0 \alpha 0} R_{\alpha 0 \beta \gamma}=\sum\left(R_{0 \alpha 0 \alpha}\right)^{2} R_{0 \alpha \beta \gamma}=0,
$$


where the sums run over the three cyclic permutations $(\alpha, \beta, \gamma)$ of $(1,2,3)$. We conclude that

$$
\sum_{\alpha=1,2,3}\left(w_{+\alpha}+w_{-\alpha}\right)^{2}\left(w_{+\alpha}-w_{-\alpha}\right)=0
$$

Substituting (3.5) into this equation yields, after some trigonometry,

$$
r^{3} \cos ^{2} \frac{\varphi_{+}-\varphi_{-}}{2} \sin \frac{\varphi_{+}-\varphi_{-}}{2} \sin \frac{3}{2}\left(\varphi_{+}+\varphi_{-}\right)=0 .
$$

Substituting the solutions

$$
r=0, \quad \varphi_{+}=\varphi_{-}+k \pi, \quad \varphi_{+}=-\varphi_{-}+k \frac{2 \pi}{3}, \quad k \in \mathbb{Z}
$$

of this equation back into (3.5) shows that the set $\left\{w_{-1}, w_{-2}, w_{-3}\right\}$ is either equal to $\left\{w_{+1}, w_{+2}\right.$, $\left.w_{+3}\right\}$ or to $\left\{-w_{+1},-w_{+2},-w_{+3}\right\}$. The first case is excluded by assumption and we conclude that $W_{+}$and $-W_{-}$have the same eigenvalues. Consequently we can choose an orthonormal basis of $V$ such that

$$
w_{+\alpha}=-w_{-\alpha}=: w_{\alpha}
$$

Then the only remaining independent components of the algebraic curvature tensor (3.6) are

$$
R_{0 \alpha \beta \gamma}=w_{\alpha} .
$$

Now consider the second integrability condition (1.7b) for $\left(a_{2}, b_{2}, c_{2}, d_{2}\right)=(0,1,2,3)$, written as

$$
\underline{a_{1}\left|b_{1}\right| c_{1} \mid d_{1}} g^{i j} g^{k l} E_{i b_{1} l d_{1}} R_{j a_{1} k c_{1}}=0
$$

with

$$
E_{i b_{1} l d_{1}}:=\frac{1}{4} \frac{\frac{a_{2}}{b_{2}}}{\frac{c_{2}}{d_{2}}} \mid R_{i b_{1} a_{2} b_{2}} R_{l d_{1} c_{2} d_{2}} .
$$

The product $R_{i b_{1} a_{2} b_{2}} R_{l d_{1} c_{2} d_{2}}$ is zero unless $\left\{i, b_{1}, a_{2}, b_{2}\right\}=\left\{l, d_{1}, c_{2}, d_{2}\right\}=\{0,1,2,3\}$. Since $\left\{a_{2}, b_{2}, c_{2}, d_{2}\right\}=\{0,1,2,3\}$, we have $E_{i b_{1} l d_{1}}=0$ unless $\left\{i, b_{1}, l, d_{1}\right\}=\{0,1,2,3\}$. The symmetries

$$
E_{b_{1} i l d_{1}}=-E_{i b_{1} l d_{1}}=E_{i b_{1} d_{1} l}, \quad E_{i b_{1} l d_{1}}=E_{l d_{1} i b_{1}}
$$

then imply that the only independent components of (3.10) are

$$
E_{0 \alpha \beta \gamma}=w_{\alpha}^{2}
$$

Now consider the full contraction of (3.9):

$$
g^{a_{1} b_{1}} g^{c_{1} d_{1}} \underline{a_{1}\left|b_{1}\right| c_{1} \mid d_{1}} E_{b_{1} d_{1}}^{i} R_{i a_{1} k c_{1}}=0 .
$$

Since the Ricci tensor of (3.8) is zero, this is results in

$$
E^{i j k l} R_{i j k l}+E^{i j k l} R_{i l k j}=0 .
$$

Using the symmetries (3.11) of $E_{i j k l}$ and those of $R_{i j k l}$, we can always permute a particular index to the first position:

$$
E^{0 j k l} R_{0 j k l}+E^{0 j k l} R_{0 l k j}=0 .
$$


And since both $E^{i j k l}$ and $R_{i j k l}$ vanish unless $\{i, j, k, l\}=\{0,1,2,3\}$, we get

$$
\sum\left(E^{0 \alpha \beta \gamma} R_{0 \alpha \beta \gamma}+E^{0 \gamma \beta \alpha} R_{0 \gamma \beta \alpha}\right)+\sum\left(E^{0 \alpha \beta \gamma} R_{0 \gamma \beta \alpha}+E^{0 \gamma \beta \alpha} R_{0 \alpha \beta \gamma}\right)=0 .
$$

Here again, the sums run over the three cyclic permutations $(\alpha, \beta, \gamma)$ of $(1,2,3)$. Substituting (3.8) and (3.12) yields

$$
\sum\left(w_{\alpha}^{3}+w_{\gamma}^{3}\right)-\sum\left(w_{\alpha}^{2} w_{\gamma}+w_{\gamma}^{2} w_{\alpha}\right)=0 .
$$

With $(2.13 \mathrm{~b})$ the second term can be transformed to

$$
\sum\left(w_{\alpha}^{2} w_{\gamma}+w_{\gamma}^{2} w_{\alpha}\right)=\sum\left(w_{\beta}^{2} w_{\alpha}+w_{\beta}^{2} w_{\gamma}\right)=\sum w_{\beta}^{2}\left(w_{\alpha}+w_{\gamma}\right)=-\sum w_{\beta}^{3},
$$

implying

$$
w_{1}^{3}+w_{2}^{3}+w_{3}^{3}=0 .
$$

By (2.13b) and Newton's identity

$$
w_{1}^{3}+w_{2}^{3}+w_{3}^{3}=3 w_{1} w_{2} w_{3}-3\left(w_{1} w_{2}+w_{2} w_{3}+w_{3} w_{1}\right)\left(w_{1}+w_{2}+w_{3}\right)+\left(w_{1}+w_{2}+w_{3}\right)^{3}
$$

this is equivalent to

$$
w_{1} w_{2} w_{3}=0 .
$$

Using (2.13b) once more, this shows that $\left\{w_{1}, w_{2}, w_{3}\right\}=\{-w, 0,+w\}$ for some $w \in \mathbb{R}$. But then (3.7) implies that $\left\{w_{+1}, w_{+2}, w_{+3}\right\}=\left\{w_{-1}, w_{-2}, w_{-3}\right\}$, which contradicts our assumption that the eigenvalues of $W_{+}$and $W_{-}$are different. Consequently $W_{+}$and $W_{-}$have the same eigenvalues. This finishes the proof of Theorem 1.13: Any integrable Killing tensor on $S^{3}$ has a diagonalisable algebraic curvature tensor.

\subsection{Solution of the second integrability condition}

It remains to prove Theorem 1.14. In the preceding subsections we showed that an algebraic curvature tensor which satisfies the algebraic integrability conditions is diagonal in a suitable orthogonal basis of $V$ and that any diagonal algebraic curvature tensor satisfies the first of the two algebraic integrability conditions. We therefore now solve the second integrability condition (1.7b) for a diagonal algebraic curvature tensor in the form (2.13). To this aim consider the tensor

$$
g^{i j} g^{k l} R_{i b_{1} a_{2} b_{2}} R_{j a_{1} k c_{1}} R_{l d_{1} c_{2} d_{2}}
$$

appearing on the left hand side of (1.7b). Suppose it does not vanish. Then we have $b_{1} \in\left\{a_{2}, b_{2}\right\}$ and $d_{1} \in\left\{c_{2}, d_{2}\right\}$, so without loss of generality we can assume $b_{1}=b_{2}$ and $d_{1}=d_{2}$. Then only terms with $i=j=a_{2}$ and $k=l=c_{2}$ contribute to the contraction and hence $\left\{a_{1}, a_{2}\right\}=$ $\left\{c_{1}, c_{2}\right\}$. If now $a_{2}=c_{2}$, then (3.13) vanishes under complete antisymmetrisation in $a_{2}, b_{2}, c_{2}$, $d_{2}$. This means that the left hand side of $(1.7 \mathrm{~b})$ vanishes unless $\left\{a_{1}, b_{1}, c_{1}, d_{1}\right\}=\left\{a_{2}, b_{2}, c_{2}, d_{2}\right\}$. Note that $a_{2}, b_{2}, c_{2}, d_{2}$ and therefore $a_{1}, b_{1}, c_{1}, d_{1}$ have to be pairwise different due to the antisymmetrisation.

For $\operatorname{dim} V=4$ in particular, (1.7b) reduces to a sole condition, which can be written as

$$
\operatorname{det}\left(\begin{array}{ccc}
1 & R_{0101}+R_{2323} & R_{0101} R_{2323} \\
1 & R_{0202}+R_{3131} & R_{0202} R_{3131} \\
1 & R_{0303}+R_{1212} & R_{0303} R_{1212}
\end{array}\right)=0 .
$$


Substituting (2.13) yields

$$
\operatorname{det}\left(\begin{array}{ccc}
1 & w_{1} & w_{1}^{2}-t_{1}^{2} \\
1 & w_{2} & w_{2}^{2}-t_{2}^{2} \\
1 & w_{3} & w_{3}^{2}-t_{3}^{2}
\end{array}\right)=0
$$

The form of this equation allows us to give an isometry invariant reformulation of the algebraic integrability conditions, which does not rely on diagonalising the algebraic curvature tensor. This also entails the isometry invariants in Proposition 1.15.

Proposition 3.4. The algebraic integrability conditions for an algebraic curvature tensor with Weyl part $\mathbf{W}$ and (not necessarily trace free) Ricci part $\mathbf{T}$ are equivalent to

$$
\begin{aligned}
& {[\mathbf{W}, \mathbf{T}]=0,} \\
& \operatorname{tr}\left(* \mathbf{W}^{2}\right)=\operatorname{tr}\left(* \mathbf{W}^{3}\right)=0
\end{aligned}
$$

and the linear dependence of $\mathbf{W}, \mathbf{W}^{2}-\mathbf{T}^{2}$ and the identity matrix.

Proof. This is a consequence of Theorem 1.13 and Proposition 2.6, together with Corollary 3.3 and the second integrability condition in the form (3.14). Indeed, (3.15a) is the definition of alignedness for $\mathbf{R}$ and $\operatorname{tr}\left(* \mathbf{W}^{k}\right)=0$ is equivalent to $\operatorname{tr} W_{+}^{k}=\operatorname{tr} W_{-}^{k}$. For $k=1$ this equation is the Bianchi identity in the form (2.2d). Hence (3.15b) means that $W_{+}$and $W_{-}$have the same characteristic polynomial. The linear dependence of $\mathbf{W}, \mathbf{W}^{2}-\mathbf{T}^{2}$ and the identity matrix is equivalent to (3.14).

For $t_{1}=t_{2}=t_{3}=0$ the left hand side of (3.14) is the Vandermonde determinant. Therefore

$$
\left(w_{1}-w_{2}\right) t_{3}^{2}+\left(w_{2}-w_{3}\right) t_{1}^{2}+\left(w_{3}-w_{1}\right) t_{2}^{2}+\left(w_{1}-w_{2}\right)\left(w_{2}-w_{3}\right)\left(w_{3}-w_{1}\right)=0 .
$$

This proves the first part of Theorem 1.14, for this equation can be written in the form

$$
\operatorname{det}\left(\begin{array}{ccc}
w_{2}-w_{3} & -t_{3} & t_{2} \\
t_{3} & w_{3}-w_{1} & -t_{1} \\
-t_{2} & t_{1} & w_{1}-w_{2}
\end{array}\right)=0 .
$$

The second part follows from the residual action of the isometry on diagonal algebraic curvature tensors as described in Section 2.5.

Regarding the eigenvalues $w_{1}, w_{2}, w_{3} \in \mathbb{R}$ as parameters subject to the restriction

$$
w_{1}+w_{2}+w_{3}=0
$$

we can solve this equation for $t_{1}, t_{2}, t_{3} \in \mathbb{R}$, depending on the number of equal eigenvalues:

1. If $w_{1}, w_{2}, w_{3}$ are pairwise different with $w_{\alpha}<w_{\beta}<w_{\gamma}$ then (3.16) describes a one sheeted hyperboloid

$$
\left(\frac{t_{\alpha}}{a_{\alpha}}\right)^{2}-\left(\frac{t_{\beta}}{a_{\beta}}\right)^{2}+\left(\frac{t_{\gamma}}{a_{\gamma}}\right)^{2}=1
$$

with semi axes

$$
\begin{aligned}
& a_{\alpha}=\sqrt{\left(w_{\beta}-w_{\alpha}\right)\left(w_{\gamma}-w_{\alpha}\right)}, \quad a_{\beta}=\sqrt{\left(w_{\beta}-w_{\alpha}\right)\left(w_{\gamma}-w_{\beta}\right)}, \\
& a_{\gamma}=\sqrt{\left(w_{\gamma}-w_{\alpha}\right)\left(w_{\gamma}-w_{\beta}\right)} .
\end{aligned}
$$


2. If $w_{\alpha}=w_{\beta} \neq w_{\gamma}$, then (3.17) implies that the solutions of (3.16) are those $\left(t_{1}, t_{2}, t_{3}\right) \in \mathbb{R}^{3}$ with $t_{\beta}= \pm t_{\alpha}$. Since $\left(t_{\alpha},-t_{\beta},-t_{\gamma}\right)$ and $\left(t_{\alpha}, t_{\beta}, t_{\gamma}\right)$ are equivalent under the isometry group, we can assume $t_{\beta}=t_{\alpha}$ without loss of generality.

3. If $w_{1}=w_{2}=w_{3}$, then (3.17) implies that $w_{1}=w_{2}=w_{3}=0$. In this case (3.16) is satisfied for any choice of $t_{1}, t_{2}, t_{3} \in \mathbb{R}$. In other words, an algebraic curvature tensor with zero Weyl component is always integrable.

\section{The algebraic geometry of the KS-variety}

We have seen that under isometries any integrable Killing tensor is equivalent to one whose algebraic curvature tensor is diagonal and that the space of such tensors is described by the following variety.

Definition 4.1. Let $\mathcal{A} \cong \mathbb{R}^{5}$ be the vector space of matrices whose symmetric part is diagonal and trace free, i.e. of matrices of the form

$$
M=\left(\begin{array}{ccc}
\Delta_{1} & -t_{3} & t_{2} \\
t_{3} & \Delta_{2} & -t_{1} \\
-t_{2} & t_{1} & \Delta_{3}
\end{array}\right)
$$

with

$$
\operatorname{tr} M=0 .
$$

We denote by $\mathcal{V} \subset \mathcal{A}$ the algebraic variety defined by the equation

$$
\operatorname{det} M=0
$$

and call its projectivisation $\mathbb{P} \mathcal{V} \subset \mathbb{P} \mathcal{A} \cong \mathbb{P}^{4}$ the Killing-Stäckel variety (KS-variety). A matrix in $\mathcal{V}$ will be called a Killing-Stäckel matrix (KS-matrix).

Remark 4.2. The residual isometry group action on diagonal algebraic curvature tensors defines a natural action of the permutation group $S_{4}$ on the Killing-Stäckel variety. This action is given by conjugation with matrices in $\mathrm{SO}(3)$ under the embedding $S_{4} \subset \mathrm{SO}(3)$ defined in Section 2.5. Later it will be useful to consider $S_{4} \subset \mathrm{SO}(3)$ as the symmetry group of an octahedron in $\mathbb{R}^{3}$ with vertices $\pm e_{i}$ and adjacent faces opposedly oriented.

The Killing-Stäckel variety $\mathbb{P} \mathcal{V}$ is a 3 -dimensional projective subvariety in $\mathbb{P}^{4}$ and the quotient $\mathbb{P} \mathcal{V} / S_{4}$ encodes all information on integrable Killing tensors modulo isometries, the metric and scalar multiples. In this section we will investigate its structure from a purely algebraic geometric point of view. This will finally lead to a complete and explicit algebraic description of Stäckel systems.

Recall that Stäckel systems are $n$-dimensional vector spaces of mutually commuting integrable Killing tensors. Note that every Stäckel system contains the metric and that the metric corresponds to the zero KS-matrix. Therefore, if we assume that the algebraic curvature tensors of the Killing tensors in a Stäckel system are mutually diagonalisable, then Stäckel systems on $S^{3}$ correspond to 2-dimensional planes in $\mathcal{V}$ or projective lines in the projectivisation $\mathbb{P} \mathcal{V}$. We will see a posteriori that our assumption is justified. Hence Stäckel systems constitute a subvariety of the variety of projective lines on $\mathbb{P} \mathcal{V}$, also called the Fano variety of $\mathbb{P} \mathcal{V}$ and denoted by $F_{1}(\mathbb{P} \mathcal{V})$.

Let $\mathcal{D}_{3}$ be the generic determinantal variety. This is the variety of $3 \times 3$ matrices with vanishing determinant or, equivalently, matrices of rank one or two. By definition $\mathbb{P} \mathcal{V}$ is a subvariety of $\mathbb{P D}_{3}$. Hence its Fano variety $F_{1}(\mathbb{P} \mathcal{V})$ is a subvariety of the Fano variety $F_{1}\left(\mathbb{P} \mathcal{D}_{3}\right)$. But the latter is well understood. It contains different types of projective linear spaces: 
1) projective spaces of matrices whose kernel contains a fixed line,

2) projective spaces of matrices whose image is contained in a fixed plane and

3) the projective plane of antisymmetric matrices.

This motivates why we seek for such spaces in $\mathbb{P} \mathcal{V}$. Note that the projective plane of antisymmetric matrices is obviously contained in $\mathbb{P} \mathcal{V}$.

Definition 4.3. We call a projective subspace in the KS-variety consisting of matrices whose kernel contains a fixed line (respectively whose image is contained in a fixed plane) an isokernel space (respectively an isoimage space).

A $3 \times 3$ matrix $M$ of rank two has a 2-dimensional image and a 1-dimensional kernel. Both are given by its adjugate matrix $\operatorname{Adj} M$, which is the transpose of the cofactor matrix and satisfies

$$
(\operatorname{Adj} M) M=M(\operatorname{Adj} M)=(\operatorname{det} M) I .
$$

The adjugate of a matrix $M \in \mathcal{V}$ is

$$
\operatorname{Adj} M=\left(\begin{array}{ccc}
t_{1} t_{1}+\Delta_{2} \Delta_{3} & t_{2} t_{1}+\Delta_{3} t_{3} & t_{3} t_{1}-\Delta_{2} t_{2} \\
t_{1} t_{2}-\Delta_{3} t_{3} & t_{2} t_{2}+\Delta_{3} \Delta_{1} & t_{3} t_{2}+\Delta_{1} t_{1} \\
t_{1} t_{3}+\Delta_{2} t_{2} & t_{2} t_{3}-\Delta_{1} t_{1} & t_{3} t_{3}+\Delta_{1} \Delta_{2}
\end{array}\right)
$$

The adjugate matrix of a rank two $3 \times 3$ matrix has rank one, which means that the columns of Adj $M$ span a line in $\mathbb{R}^{3}$. And because $\operatorname{det} M=0$, we deduce from (4.2) that the kernel of $M$ is the column space of $\operatorname{Adj} M$.

The adjugate matrix of a rank one $3 \times 3$ matrix is zero. It is known, that the singular locus of $\mathbb{P D}_{3}$ is the subvariety of rank one matrices. The following proposition characterises the singular locus of the KS-variety. For simplicity we will be a bit imprecise and not strictly distinguish between a matrix $M \in \mathcal{V} \backslash\{0\}$ and its image $\mathbb{R} M \in \mathbb{P} \mathcal{V}$ under the canonical projection $\mathcal{V} \backslash\{0\} \rightarrow \mathbb{P} \mathcal{V}$. Depending on the context, we will refer to an element of $\mathbb{P} \mathcal{V}$ as "matrix" or "point".

Proposition 4.4 (singularities of the KS-variety). The $K S$-variety $\mathbb{P} \mathcal{V}$ has ten singular points, given by the six rank one matrices

$$
V_{ \pm 1}:=\left(\begin{array}{ccc}
0 & 0 & 0 \\
0 & +1 & \mp 1 \\
0 & \pm 1 & -1
\end{array}\right), \quad V_{ \pm 2}:=\left(\begin{array}{ccc}
-1 & 0 & \pm 1 \\
0 & 0 & 0 \\
\mp 1 & 0 & +1
\end{array}\right), \quad V_{ \pm 3}:=\left(\begin{array}{ccc}
+1 & \mp 1 & 0 \\
\pm 1 & -1 & 0 \\
0 & 0 & 0
\end{array}\right)
$$

and the four skew symmetric matrices

$$
C_{0}:=V_{+1}+V_{+2}+V_{+3}, \quad C_{\alpha}:=V_{-\alpha}+V_{+\beta}+V_{+\gamma},
$$

where $(\alpha, \beta, \gamma)$ denotes an even permutation of $(1,2,3)$. Moreover, the rank one matrices in $\mathbb{P} \mathcal{V}$ are exactly those six in (4.4a).

Definition 4.5. We will call the six singularities $V_{ \pm \alpha}$ the rank one singular points in $\mathbb{P} \mathcal{V}$ and the four singularities $C_{i}$ the skew symmetric singular points in $\mathbb{P} \mathcal{V}$.

Proof. $\mathbb{P} \mathcal{V}$ is singular at $\mathbb{R} M$ if and only if $\mathcal{V}$ is singular at $M$. Since $\mathcal{V}$ is the zero locus of the determinant function det: $\mathcal{A} \rightarrow \mathbb{R}$, this is the case if and only if the derivative of the determinant function at $M \in \mathcal{V}$, given by

$$
\begin{aligned}
(d \operatorname{det})_{M}: & \mathcal{A} \\
A & \mapsto \mathbb{R}, \\
A & \mapsto \operatorname{tr}(A \operatorname{Adj} M),
\end{aligned}
$$


is the zero map. The condition that $\operatorname{tr}(A \operatorname{Adj} M)=0$ for all $A \in \mathcal{A}$ is equivalent to

$$
t_{1}^{2}+\Delta_{2} \Delta_{3}=t_{2}^{2}+\Delta_{3} \Delta_{1}=t_{3}^{2}+\Delta_{1} \Delta_{2}, \quad \Delta_{1} t_{1}=\Delta_{2} t_{2}=\Delta_{3} t_{3}=0 .
$$

We leave it to the reader to verify that the solutions $M \in \mathcal{V}$ of these equations are exactly the matrices (4.4) and their multiples.

The last statement now follows from (4.5) and the equivalence of $\operatorname{rank} M=1$ and $\operatorname{Adj} M=0$ for $3 \times 3$-matrices.

Remark 4.6. Under the natural $S_{4}$-action on $\mathbb{P} \mathcal{V}$, all rank one singular points are equivalent. This means that the singularities $V_{ \pm \alpha}$ are all mapped to a single point $V$ in the quotient $\mathbb{P} \mathcal{V} / S_{4}$. The same holds for the skew symmetric singular points, which are mapped to a single point $C \in$ $\mathbb{P} \mathcal{V} / S_{4}$.

We now compute the isokernel spaces in $\mathbb{P} \mathcal{V}$. To find all matrices $M \in \mathcal{V}$ annihilating a given vector $\vec{n} \in \mathbb{R}^{3}$, we consider the equation $M \vec{n}=0$ as an equation in $M$ for a fixed $\vec{n}=\left(n_{1}, n_{2}, n_{3}\right)$, where $M$ is of the form $M=\Delta+T$ with

$$
\Delta=\left(\begin{array}{ccc}
\Delta_{1} & 0 & 0 \\
0 & \Delta_{2} & 0 \\
0 & 0 & \Delta_{3}
\end{array}\right), \quad T=\left(\begin{array}{ccc}
0 & -t_{3} & t_{2} \\
t_{3} & 0 & -t_{1} \\
-t_{2} & t_{1} & 0
\end{array}\right)
$$

We can regard $\vec{t}=\left(t_{1}, t_{2}, t_{3}\right) \in \mathbb{R}^{3}$ as a parameter and solve $\Delta \vec{n}=-T \vec{n}$ for $\Delta$. For the time being, let us assume $n_{1} n_{2} n_{3} \neq 0$. We then obtain a linear family of matrices

$$
M=\left(\begin{array}{ccc}
\frac{n_{2}}{n_{1}} t_{3}-\frac{n_{3}}{n_{1}} t_{2} & -t_{3} & t_{2} \\
t_{3} & \frac{n_{3}}{n_{2}} t_{1}-\frac{n_{1}}{n_{2}} t_{3} & -t_{1} \\
-t_{2} & t_{1} & \frac{n_{1}}{n_{3}} t_{2}-\frac{n_{2}}{n_{3}} t_{1}
\end{array}\right) .
$$

All these matrices satisfy $\operatorname{det} M=0$ and the condition $\operatorname{tr} M=0$ imposes the restriction

$$
\vec{t} \perp\left(\frac{n_{3}}{n_{2}}-\frac{n_{2}}{n_{3}}, \frac{n_{1}}{n_{3}}-\frac{n_{3}}{n_{1}}, \frac{n_{2}}{n_{1}}-\frac{n_{1}}{n_{2}}\right)
$$

on the parameter $\vec{t} \in \mathbb{R}^{3}$. This shows that the matrices in $\mathcal{V}$ annihilating a fixed vector $\vec{n} \neq 0$ form a linear subspace. If the right hand side of (4.6b) is zero, i.e. if the condition is void, this subspace has dimension three and defines a projective plane in $\mathbb{P} \mathcal{V}$. This happens if and only if

$$
\left|n_{1}\right|=\left|n_{2}\right|=\left|n_{3}\right| \text {. }
$$

If the right hand side of $(4.6 \mathrm{~b})$ is not zero, this subspace has dimension two and defines a projective line in $\mathbb{P} \mathcal{V}$. One can check that this also holds true for the case $n_{1} n_{2} n_{3}=0$. We have shown the following:

Lemma 4.7. If $\vec{n} \in \mathbb{R}^{3}$ satisfies (4.7), then the set of matrices in $\mathbb{P} \mathcal{V}$ annihilating $\vec{n}$ is a projective plane of the form (4.6a). Otherwise it is a projective line.

Recall that the kernel of a rank two matrix of the form (4.1) is the row space of the adjugate matrix (4.3). This defines a rational map

$$
\pi: \mathbb{P} \mathcal{V} \rightarrow \mathbb{P}^{2},
$$

whose fibres are the maximal isokernel spaces. This map is well defined except for the six rank one matrices in $\mathbb{P} \mathcal{V}$. 
We want to give a parametrisation of the isokernel lines that is uniform and more geometric than (4.6). For this purpose we define two embeddings $\iota, \nu: \mathbb{P}^{2} \rightarrow \mathbb{P} \mathcal{V}$ given by

$$
\iota(n)=\left(\begin{array}{ccc}
0 & -n_{3} & n_{2} \\
n_{3} & 0 & -n_{1} \\
-n_{2} & n_{1} & 0
\end{array}\right), \quad \nu(n)=\left(\begin{array}{ccc}
n_{2}^{2}-n_{3}^{2} & -n_{1} n_{2} & n_{3} n_{1} \\
n_{1} n_{2} & n_{3}^{2}-n_{1}^{2} & -n_{2} n_{3} \\
-n_{3} n_{1} & n_{2} n_{3} & n_{1}^{2}-n_{2}^{2}
\end{array}\right)
$$

for $n=\left(n_{1}: n_{2}: n_{3}\right) \in \mathbb{P}^{2}$. One readily checks that $\iota(n)$ and $\nu(n)$ indeed lie in $\mathbb{P} \mathcal{V}$. Note that the image of $\iota$ is the projective plane of skew symmetric matrices in $\mathbb{P} \mathcal{V}$. Under the inclusion $\mathbb{P} \mathcal{V} \subset \mathbb{P} \mathcal{A} \cong \mathbb{P}^{4}$ the map $\iota$ is a linear embedding of $\mathbb{P}^{2}$ in $\mathbb{P}^{4}$ whilst $\nu$ is the composition of the Veronese embedding

$$
\begin{aligned}
\mathbb{P}^{2} & \hookrightarrow \mathbb{P}^{5}, \\
\left(n_{1}: n_{2}: n_{3}\right) & \mapsto\left(n_{1}^{2}: n_{2}^{2}: n_{3}^{2}: n_{2} n_{3}: n_{3} n_{1}: n_{1} n_{2}\right),
\end{aligned}
$$

and a projection $\mathbb{P}^{5} \rightarrow \mathbb{P}^{4}$ under which the Veronese surface remains smooth.

Proposition 4.8 (isokernel spaces in the KS-variety). Recall from Proposition 4.4 that the singular points in the $K S$-variety $\mathbb{P} \mathcal{V}$ are the four skew symmetric singular points $C_{0}, C_{1}, C_{2}$, $C_{3}$ as well as six rank one singular points $V_{ \pm \alpha}, \alpha=1,2,3$.

1. The projective plane through $V_{+1}, V_{+2}$ and $V_{+3}$ is an isokernel plane in $\mathbb{P} \mathcal{V}$ and contains $C_{0}$. In the same way, the three points $V_{-\alpha}, V_{+\beta}$ and $V_{+\gamma}$ define an isokernel plane through $C_{\alpha}$ for $\alpha=1,2,3$.

Let the maps $\pi: \mathbb{P} \mathcal{V} \rightarrow \mathbb{P}^{2}$ and $\iota, \nu: \mathbb{P}^{2} \rightarrow \mathbb{P} \mathcal{V}$ be defined as above.

2. For any non-singular point $M \in \mathbb{P} \mathcal{V}$, the points $M, \iota(\pi(M))$ and $\nu(\pi(M))$ are collinear but do not all three coincide. The projective line they define lies in $\mathbb{P} \mathcal{V}$ and is an isokernel space through $M$. Each of these lines contains a unique skew symmetric point, namely $\iota(\pi(M))$.

Moreover, any isokernel space in $\mathbb{P} \mathcal{V}$ of maximal dimension is of either of the above forms and hence contains a unique skew symmetric point.

Remark 4.9. A corresponding characterisation of isoimage spaces follows from the fact that matrix transposition defines an involution of $\mathbb{P} \mathcal{V}$ which interchanges isokernel and isoimage spaces.

Proof. 1 follows directly from Lemma 4.7 and the explicit form (4.4) of the singular points. For 2 notice that $n:=\pi(M)$ is the kernel of $M$ and well defined for $M \neq V_{\alpha}$. The definitions (4.8) of $\iota$ and $\nu$ then show that $n$ is also the kernel of $\iota(n)$ and $\nu(n)$. These definitions also show that $M, \iota(n)$ and $\nu(n)$ do not all coincide for $M \neq C_{i}$. Their collinearity follows from Lemma 4.7 in the case where $n$ does not satisfy $(4.7)$ and from $\iota(n)=\nu(n)$ in case it does. The last statement is now a consequence of Lemma 4.7 .

We can illustrate the content of Proposition 4.8 geometrically as follows. This is depicted in Fig. 1. The six rank one singular points of $\mathbb{P} \mathcal{V} \subset \mathbb{P}^{4}$ constitute the vertices of a regular octahedron in $\mathbb{P}^{4}$ whose faces (and the planes they define) are entirely contained in $\mathbb{P} \mathcal{V}$. The set of eight faces is divided into two sets of non-adjacent faces, corresponding to the four isokernel planes (shaded) respectively the four isoimage planes in $\mathbb{P} \mathcal{V}$. Opposite faces intersect in their respective centres. These are the skew symmetric singular points of $\mathbb{P} \mathcal{V}$ and the intersection points of the octahedron with the projective plane of skew symmetric matrices. In Fig. 1 this ninth projective plane in $\mathbb{P} \mathcal{V}$ is depicted as the insphere of the octahedron. 


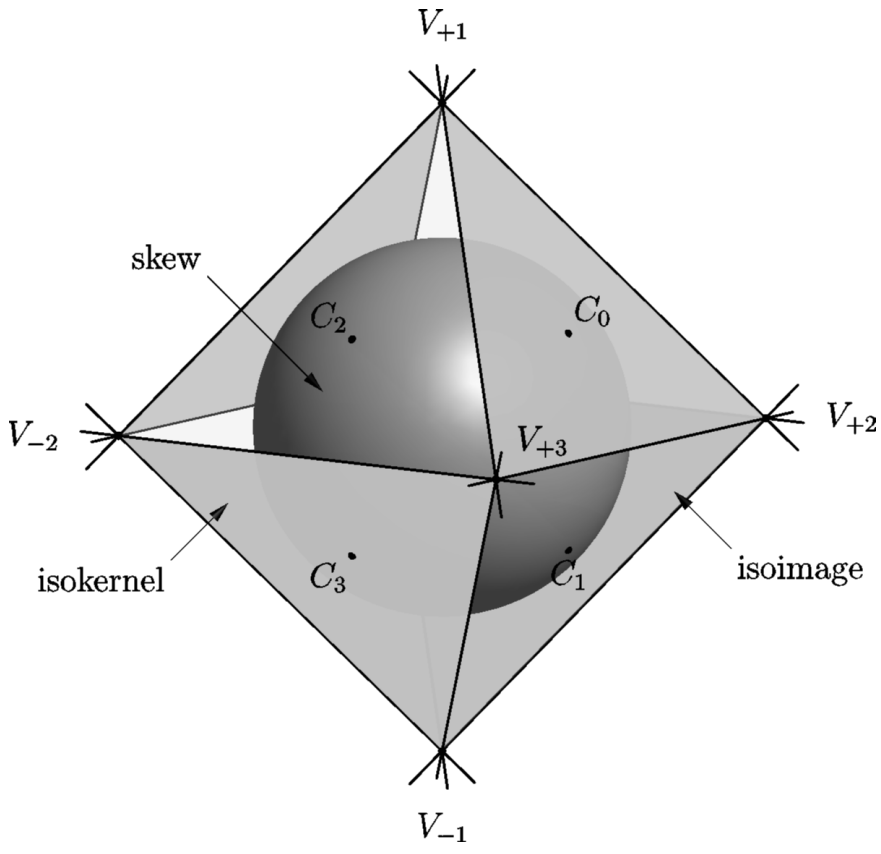

Figure 1. Singularities and projective planes in the KS-variety.

The permutation group $S_{4}$ acts on this configuration by symmetries of the octahedron preserving the two sets of non-adjacent faces. Matrix transposition corresponds to a point reflection, which exchanges opposite faces and completes the $S_{4}$-action to the full octahedral symmetry.

In anticipation of the results in the next section we introduce the following name:

Definition 4.10. We call the isokernel lines of the form 2 in Proposition 4.8 "Stäckel lines".

Every Stäckel line intersects the projective plane of skew symmetric matrices transversely. Conversely, every point on this plane determines a unique Stäckel line unless it is one of the four singular skew symmetric points. The Stäckel lines through these points are exactly those projective lines in each isokernel face which pass through the respective face center.

\section{$5 \quad$ Stäckel systems}

In this section we express the condition that two Killing tensors commute as an algebraic condition on the corresponding algebraic curvature tensors. This will eventually justify the term "Stäckel lines" chosen in Definition 4.10 by proving that these lines indeed correspond to Stäckel systems.

Remark 5.1. Most results in this and the following section are valid for an arbitrary non-flat constant curvature (pseudo-)Riemannian manifold $M$ that is embedded into some vector space $V$ as the hypersurface

$$
M=\{v \in V: g(v, v)=1\} \subset V
$$

of length one vectors with respect to a non-degenerated symmetric bilinear form $g$ on $V$. Note that any non-flat constant curvature (psudo-)Riemannian manifold is locally isometric to such a model. 


\subsection{Young tableaux}

Throughout this section we use Young tableaux in order to characterise index symmetries of tensors. Young tableaux define elements in the group algebra of the permutation group $S_{d}$. That is, a Young tableau stands for a (formal) linear combination of permutations of $d$ objects. In our case, these objects will be certain tensor indices. For the sake of simplicity of notation we will identify a Young tableau with the group algebra element it defines. It will be sufficient for our purposes to consider the examples below. For more examples and a detailed explanation of the notation as well as the techniques used here, we refer the reader to [41].

A Young tableau consisting of a single row denotes the sum of all permutations of the indices in this row. For example, using cycle notation,

$$
\underline{a_{2}\left|c_{1}\right| c_{2}}=e+\left(a_{2} c_{1}\right)+\left(c_{1} c_{2}\right)+\left(c_{2} a_{2}\right)+\left(a_{2} c_{1} c_{2}\right)+\left(c_{2} c_{1} a_{2}\right) .
$$

This is an element in the group algebra of the group of permutations of the indices $a_{2}, c_{1}$ and $c_{2}$ (or any superset). In the same way a Young tableau consisting of a single column denotes the signed sum of all permutations of the indices in this column, the sign being the sign of the permutation. For example,

$$
\begin{aligned}
& \frac{a_{1}}{b_{1}} \\
& \hline d_{2}
\end{aligned}=e-\left(a_{1} b_{1}\right)-\left(b_{1} d_{2}\right)-\left(d_{2} a_{1}\right)+\left(a_{1} b_{1} d_{2}\right)+\left(d_{2} b_{1} a_{1}\right) .
$$

We call these row symmetrisers respectively column antisymmetrisers. The reason we define them without the usual normalisation factors is that then all numerical constants appear explicitly in our computations (although irrelevant for our concerns).

The group multiplication extends linearly to a natural product in the group algebra. A general Young tableau is then simply the product of all column antisymmetrisers and all row symmetrisers of the tableau. We will only deal with Young tableaux having a "hook shape", such as the following:

$$
\begin{array}{|l|l|l|}
\hline a_{1}\left|a_{2}\right| c_{1} \mid c_{2} \\
\hline b_{1} & =a_{a_{1}}\left|a_{2}\right| c_{1} \mid c_{2} & \frac{a_{1}}{b_{1}} \\
\hline d_{2} & \\
\hline d_{2} \\
\hline
\end{array} .
$$

The inversion of group elements extends linearly to an involution of the group algebra. If we consider elements in the group algebra as linear operators on the group algebra itself, this involution is the adjoint with respect to the natural inner product on the group algebra, given by defining the group elements to be an orthonormal basis. Since this operation leaves symmetrisers and antisymmetrisers invariant, it simply exchanges the order of symmetrisers and antisymmetrisers in a Young tableau. The adjoint of (5.1) for example is

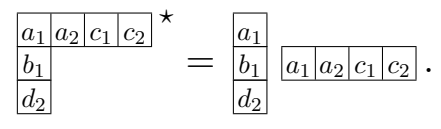

Properly scaled, Young tableaux define projectors onto irreducible $S_{d}$-representations. We are now able to state a lemma which expresses the decomposition

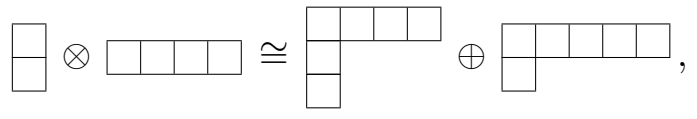

given by the Littlewood-Richardson rule, in terms of Young projectors. Its proof can be found in [41]. 
Lemma 5.2. In the group algebra of the permutation group of the indices $a_{1}, a_{2}, c_{1}, c_{2}, b_{1}$ and $d_{2}$ the following identity holds:

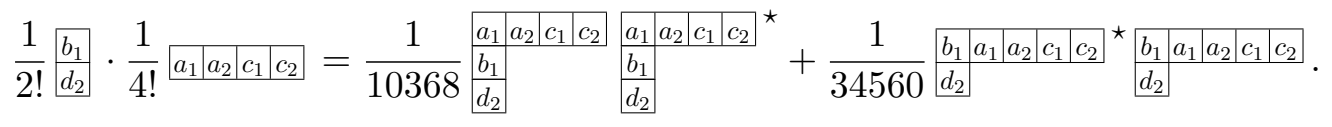

The action of the permutation group on tensors extends linearly to an action of the group algebra. In particular, any Young tableau acts on tensors with corresponding indices. For example,

$$
\begin{array}{|l|l}
\frac{b_{1}}{a_{2}} \\
\hline c_{2}
\end{array} T_{b_{1} a_{2} c_{2}}=T_{b_{1} a_{2} c_{2}}-T_{a_{2} b_{1} c_{2}}-T_{b_{1} c_{2} a_{2}}-T_{c_{1} a_{2} b_{2}}+T_{a_{1} c_{2} b_{2}}+T_{c_{1} b_{2} a_{2}} .
$$

To give another example, the operator (5.1) acts on a tensor $T_{b_{1} b_{2} d_{1} d_{2} a_{2} c_{2}}$ by an antisymmetrisation in the indices $b_{1}, a_{2}, c_{2}$ and a subsequent symmetrisation in the indices $b_{1}, b_{2}, d_{1}, d_{2}$. In the same way its adjoint (5.2) acts by first symmetrising and then antisymmetrising.

\subsection{Commuting Killing tensors}

In the following we express the fact that two Killing tensors commute (as endomorphisms) as a purely algebraic condition on their corresponding algebraic curvature tensors. Notice that the commuting of Killing tensors as endomorphisms on the tangent space is not equivalent to the commuting of their respective algebraic curvature tensors as endomorphisms on $\Lambda^{2} V$, as one may guess naïvely.

Proposition 5.3 (algebraic counterpart of the commutator). Let $K$ and $\tilde{K}$ be two Killing tensors on a non-flat constant curvature manifold with algebraic curvature tensors $R$ and $\tilde{R}$. Then the following statements are equivalent

$$
\begin{aligned}
& {[K, \tilde{K}]=0,}
\end{aligned}
$$

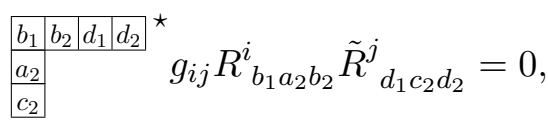

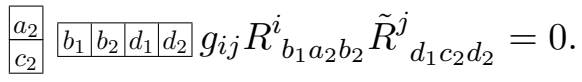

Proof. Using $\nabla_{v} x^{b}=v^{b}$ we write (1.6) in local coordinates for coordinate vectors $v=\partial_{\alpha}$ and $w=\partial_{\beta}$ :

$$
K_{\alpha \beta}=R_{a_{1} b_{1} a_{2} b_{2}} x^{a_{1}} x^{a_{2}} \nabla_{\alpha} x^{b_{1}} \nabla_{\beta} x^{b_{2}} .
$$

The product of $K$ and $\tilde{K}$, regarded as endomorphisms, is then given by

$$
K_{\gamma}^{\alpha} \tilde{K}_{\beta}^{\gamma}=R_{a_{1} b_{1} a_{2} b_{2}} \tilde{R}_{c_{1} d_{1} c_{2} d_{2}} x^{a_{1}} x^{a_{2}} x^{c_{1}} x^{c_{2}} \nabla^{\alpha} x^{b_{1}} \nabla_{\gamma} x^{b_{2}} \nabla^{\gamma} x^{d_{1}} \nabla_{\beta} x^{d_{2}} .
$$

In [41] we proved the following identity:

$$
\nabla_{\gamma} x^{b_{2}} \nabla^{\gamma} x^{d_{1}}=g^{b_{2} d_{1}}-x^{b_{2}} x^{d_{1}} .
$$

As a consequence of the antisymmetry of algebraic curvature tensors in the last index pair, the term $x^{b_{2}} x^{d_{1}}$ does not contribute when substituting this identity into the previous expression:

$$
K_{\gamma}^{\alpha} \tilde{K}_{\beta}^{\gamma}=g^{b_{2} d_{1}} R_{a_{1} b_{1} a_{2} b_{2}} \tilde{R}_{c_{1} d_{1} c_{2} d_{2}} x^{a_{1}} x^{a_{2}} x^{c_{1}} x^{c_{2}} \nabla^{\alpha} x^{b_{1}} \nabla_{\beta} x^{d_{2}} .
$$


The commutator $[K, \tilde{K}]$ is therefore given by

$$
[K, \tilde{K}]_{\alpha \beta}=g^{b_{2} d_{1}} R_{a_{1} b_{1} a_{2} b_{2}} \tilde{R}_{c_{1} d_{1} c_{2} d_{2}} x^{a_{1}} x^{a_{2}} x^{c_{1}} x^{c_{2}} \nabla_{[\alpha} x^{b_{1}} \nabla_{\beta]} x^{d_{2}}
$$

and vanishes if and only if

$$
g^{b_{2} d_{1}} R_{a_{1} b_{1} a_{2} b_{2}} \tilde{R}_{c_{1} d_{1} c_{2} d_{2}} x^{a_{1}} x^{a_{2}} x^{c_{1}} x^{c_{2}} v^{\left[b_{1}\right.} w^{\left.d_{2}\right]}=0
$$

for all $x \in M$ and $v, w \in T_{x} M$. That is, for all $x, v, w \in V$ with

$$
g(x, x)=1, \quad g(x, v)=g(x, w)=0 .
$$

We can drop the restriction $g(x, x)=1$ since $\mathbb{R} M \subseteq V$ is open. We can also drop the restrictions $g(x, v)=0$ and $g(x, w)=0$ by decomposing arbitrary vectors $v, w \in V$ under the decomposition $V=T_{x} M \oplus \mathbb{R} x$. To see this, notice that (5.5) is trivially satisfied for $v=x$ or for $w=x$. Indeed, in this case the tensor

$$
R_{a_{1} b_{1} a_{2} b_{2}} \tilde{R}_{c_{1} d_{1} c_{2} d_{2}}
$$

is implicitly symmetrised over five indices and Dirichlet's drawer principle tells us that this comprises a symmetrisation in one of the four antisymmetric index pairs. This means we can omit the restrictions (5.6) completely. In other words, $[K, \tilde{K}]=0$ is equivalent to (5.5) being satisfied for all $x, v, w \in V$.

Now notice that the tensor

$$
x^{a_{1}} x^{a_{2}} x^{c_{1}} x^{c_{2}} v^{\left[b_{1}\right.} w^{\left.d_{2}\right]}
$$

appearing in (5.5) is completely symmetric in the indices $a_{1}, a_{2}, c_{1}$ and $c_{2}$ and completely antisymmetric in the indices $b_{1}$ and $d_{2}$. Applying (5.3) to this tensor therefore yields

$$
\begin{aligned}
& x^{a_{1}} x^{a_{2}} x^{c_{1}} x^{c_{2}} v^{\left[b_{1}\right.} w^{\left.d_{2}\right]}=\frac{1}{2 !} \frac{b_{1}}{d_{2}} \cdot \frac{1}{4 !} \stackrel{a_{1}\left|a_{2}\right| c_{1} \mid c_{2}}{\underline{a_{2}}} x^{a_{1}} x^{a_{2}} x^{c_{1}} x^{c_{2}} v^{\left[b_{1}\right.} w^{\left.d_{2}\right]}
\end{aligned}
$$

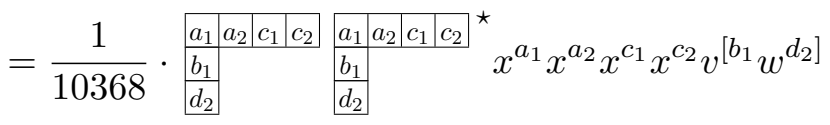

$$
\begin{aligned}
& +\left.\frac{1}{34560} \cdot \frac{b_{1}\left|a_{1}\right| a_{2}\left|c_{1}\right| c_{2}}{d_{2} \mid}\right|^{\star} \frac{b_{1}\left|a_{1}\right| a_{2}\left|c_{1}\right| c_{2}}{d_{2}} x^{a_{1}} x^{a_{2}} x^{c_{1}} x^{c_{2}} v^{\left[b_{1}\right.} w^{\left.d_{2}\right]} .
\end{aligned}
$$

From the definition of the Young tableaux and their adjoints,

$$
\begin{aligned}
& \begin{array}{|l|l|l|l|}
\hline \frac{a_{1}}{a_{2}}\left|c_{1}\right| c_{2} \\
\hline b_{1} \\
\hline d_{2}
\end{array}=a_{a_{1}\left|a_{2}\right| c_{1} \mid c_{2}} \begin{array}{|l}
\frac{a_{1}}{b_{1}} \\
\frac{d_{2}}{2}
\end{array},
\end{aligned}
$$

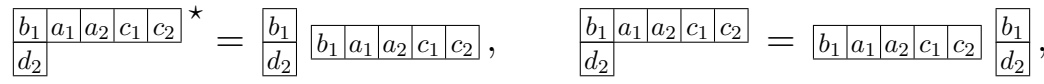

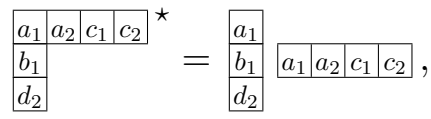

together with the properties

$$
\underline{a_{1}\left|a_{2}\right| c_{1}\left|c_{2}\right|} x^{a_{1}} x^{a_{2}} x^{c_{1}} x^{c_{2}}=4 ! x^{a_{1}} x^{a_{2}} x^{c_{1}} x^{c_{2}}, \quad\left[\frac{b_{1}}{d_{2}}\left|v^{\left[b_{1}\right.} w^{\left.d_{2}\right]}=2 \frac{b_{1}}{d_{2}}\right| v^{b_{1}} w^{d_{2}}\right.
$$

and

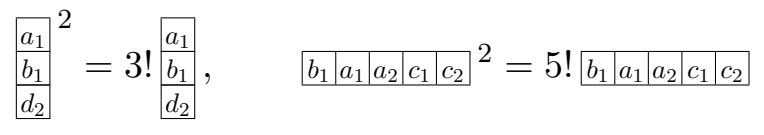


we see that (5.8) simplifies to

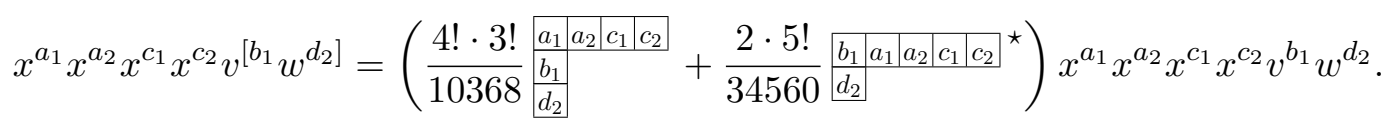

When substituted into (5.5), this yields

$$
g^{b_{2} d_{1}} R_{a_{1} b_{1} a_{2} b_{2}} \tilde{R}_{c_{1} d_{1} c_{2} d_{2}}\left(\frac{1}{72} \frac{{\frac{a_{1}}{b_{1}}\left|a_{2}\right| c_{1} \mid c_{2}}_{d_{2} \mid} \mid}{d^{2}}+\frac{1}{144}{\frac{b_{1}\left|a_{1}\right| a_{2}\left|c_{1}\right| c_{2}}{d_{2} \mid}}^{\star}\right) x^{a_{1}} x^{a_{2}} x^{c_{1}} x^{c_{2}} v^{b_{1}} w^{d_{2}}=0 .
$$

As elements of the group algebra of the permutation group, the Young tableaux are linear combinations of permutations $\pi$ of the indices $a_{1}, a_{2}, c_{1}, c_{2}, b_{1}, d_{2}$. For any such permutation $\pi$ we have

$$
\begin{aligned}
& g^{b_{2} d_{1}} R_{a_{1} b_{1} a_{2} b_{2}} \tilde{R}_{c_{1} d_{1} c_{2} d_{2}} x^{\pi\left(a_{1}\right)} x^{\pi\left(a_{2}\right)} x^{\pi\left(c_{1}\right)} x^{\pi\left(c_{2}\right)} v^{\pi\left(b_{1}\right)} w^{\pi\left(d_{2}\right)} \\
& \quad=g^{b_{2} d_{1}} R_{\pi^{\star}\left(a_{1}\right) \pi^{\star}\left(b_{1}\right) \pi^{\star}\left(a_{2}\right) b_{2}} \tilde{R}_{\pi^{\star}\left(c_{1}\right) d_{1} \pi^{\star}\left(c_{2}\right) \pi^{\star}\left(d_{2}\right)} x^{a_{1}} x^{a_{2}} x^{c_{1}} x^{c_{2}} v^{b_{1}} w^{d_{2}},
\end{aligned}
$$

where $\pi^{\star}=\pi^{-1}$ is the adjoint of $\pi$. We can thus replace the Young tableaux in (5.9) acting on upper indices by its adjoint acting on lower indices:

$$
\left(\left(\frac{1}{72} \frac{a_{1}^{a_{1}\left|a_{2}\right| c_{1} \mid c_{2}}}{{\underline{b_{2}}}^{d_{2}}}{ }^{\star}+\frac{1}{144} \frac{\underline{b}_{1}\left|a_{1}\right| a_{2}\left|c_{1}\right| c_{2}}{d_{2}}\right) g^{b_{2} d_{1}} R_{a_{1} b_{1} a_{2} b_{2}} \tilde{R}_{c_{1} d_{1} c_{2} d_{2}}\right) x^{a_{1}} x^{a_{2}} x^{c_{1}} x^{c_{2}} v^{b_{1}} w^{d_{2}}=0 .
$$

Now notice that the second Young tableau involves a symmetrisation over the five indices $b_{1}$, $a_{1}, a_{2}, c_{1}, c_{2}$ and that, as above, the symmetrisation of the tensor (5.7) in any five indices is zero. Hence the second term in (5.10) vanishes and we obtain

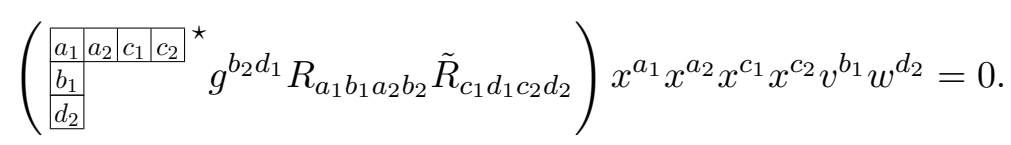

Recall that $[K, \tilde{K}]=0$ is equivalent to this condition being satisfied for all $x, v, w \in V$. By polarising in $x$ we get

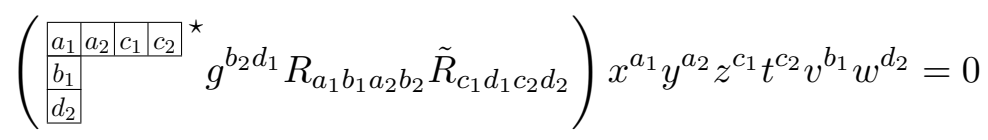

for all $x, y, z, t, v, w \in V$ and hence

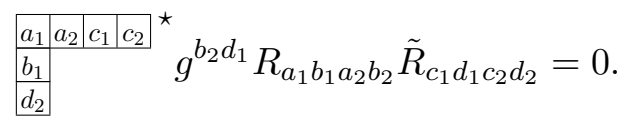

This is the same as (5.4b) after appropriately renaming, lowering and rising indices. We have proven the equivalence $(5.4 \mathrm{a}) \Leftrightarrow(5.4 \mathrm{~b})$.

We now prove the equivalence $(5.4 \mathrm{~b}) \Leftrightarrow(5.4 \mathrm{c})$. Start from $(5.4 \mathrm{~b})$ by expanding the Young tableau:

$$
\begin{array}{|l|l|l|l}
\hline \frac{b_{1}}{a_{2}} \\
\hline c_{2} \\
\hline b_{1}\left|b_{2}\right| d_{1} \mid d_{2}
\end{array} g_{i j} R_{b_{1} a_{2} b_{2}}^{i} \tilde{R}_{d_{1} c_{2} d_{2}}^{j}=0 .
$$

In order to sum over all 4 ! permutations when carrying out the symmetrisation in the indices $b_{1}, b_{2}, d_{1}, d_{2}$, one can first take the sum over the 4 cyclic permutations of $b_{1}, b_{2}, d_{1}, d_{2}$, then fix the index $b_{1}$ and finally sum over all 3 ! permutations of the remaining 3 indices $b_{2}, d_{1}, d_{2}$ :

$$
\begin{array}{|l}
\frac{b_{1}}{a_{2}} \\
\hline c_{2}
\end{array} \underline{b_{2}\left|d_{1}\right| d_{2}} g_{i j}\left(R_{\underline{b}_{1} \underline{a}_{2} b_{2}}^{i} \tilde{R}_{d_{1} \underline{c}_{2} d_{2}}^{j}+R_{b_{2} \underline{a}_{2} d_{1}}^{i} \tilde{R}_{d_{2} \underline{\underline{c}}_{2} \underline{b}_{1}}^{j}+R_{d_{1} \underline{a}_{2} d_{2}}^{i} \tilde{R}_{\underline{b}_{1} \underline{c}_{2} b_{2}}^{j}+R_{d_{2} \underline{a}_{2} \underline{b}_{1}}^{i} \tilde{R}_{b_{2} \underline{c}_{2} d_{1}}^{j}\right)=0 .
$$


For a better readability we underlined each antisymmetrised index. Permuting the indices of the terms in the parenthesis under symmetrisation in $b_{2}, d_{1}, d_{2}$ and antisymmetrisation in $b_{1}$, $a_{2}, c_{2}$ we can gather the first and last as well as the second and third term:

$$
\left.\begin{array}{|c|}
\hline \frac{b_{1}}{a_{2}} \\
\hline c_{2}
\end{array}\right] \underline{b_{2}\left|d_{1}\right| d_{2}} g_{i j}\left(\left(R_{\underline{b}_{1} \underline{a}_{2} b_{2}}^{i}+R_{b_{2} \underline{a}_{2} \underline{b}_{1}}^{i}\right) \tilde{R}_{d_{1} \underline{c}_{2} d_{2}}^{j}+R_{d_{1} \underline{a}_{2} d_{2}}^{i}\left(\tilde{R}_{\underline{b}_{1} \underline{c}_{2} b_{2}}^{j}+\tilde{R}_{b_{2} \underline{c}_{2} \underline{b}_{1}}^{j}\right)\right)=0 .
$$

Using the symmetries of $R_{b_{1} a_{2} b_{2}}$, the terms in the inner parentheses can be rewritten as

$$
R_{\underline{\underline{b}}_{1} \underline{a}_{2} b_{2}}^{i}=-R_{\underline{\underline{b}}_{1} b_{2} \underline{a}_{2}}^{i}, \quad R_{b_{2} \underline{a}_{2} \underline{\underline{b}}_{1}}^{i}=-R_{\underline{a}_{2} \underline{b}_{1} b_{2}}^{i}-R_{\underline{b}_{1} b_{2} \underline{a}_{2}}^{i}=R_{\underline{a}_{2} b_{2} \underline{b}_{1}}^{i}-R_{\underline{b}_{1} b_{2} \underline{a}_{2}}^{i}
$$

resulting in

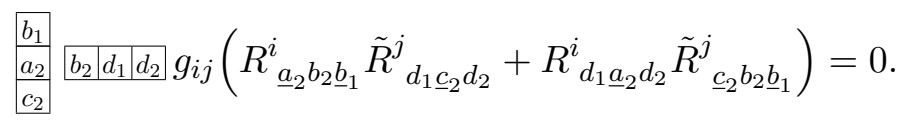

As above, when carrying out the antisymmetrisation over $b_{1}, a_{2}, c_{2}$, we can first sum over the three cyclic permutations of $b_{1}, a_{2}, c_{2}$, then fix $b_{1}$ and finally sum over the two permutations of $a_{2}, c_{2}$. This results in

$$
\begin{gathered}
\qquad \frac{a_{2}}{c_{2}} \sqrt{b_{2}\left|d_{1}\right| d_{2}} g_{i j}\left(R_{a_{2} b_{2} b_{1}}^{i} \tilde{R}_{d_{1} c_{2} d_{2}}^{j}+R_{d_{1} a_{2} d_{2}}^{i} \tilde{R}_{c_{2} b_{2} b_{1}}^{j}+R_{c_{2} b_{2} a_{2}}^{i} \tilde{R}_{d_{1} b_{1} d_{2}}^{j}+R_{d_{1} c_{2} d_{2}}^{i} \tilde{R}_{b_{1} b_{2} a_{2}}^{j}\right. \\
\left.+R_{b_{1} b_{2} c_{2}}^{i} \tilde{R}_{d_{1} a_{2} d_{2}}^{j}+R_{d_{1} b_{1} d_{2}}^{i} \tilde{R}_{a_{2} b_{2} c_{2}}^{j}\right)=0 .
\end{gathered}
$$

The symmetrisation of this in $b_{1}, b_{2}, d_{1}, d_{2}$ yields

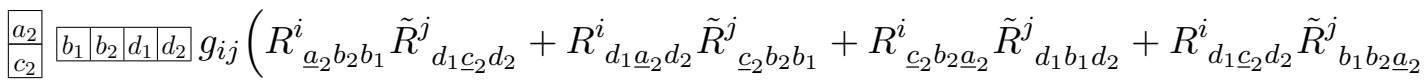

$$
\begin{aligned}
& \left.+R_{b_{1} b_{2} \underline{c}_{2}}^{i} \tilde{R}_{d_{1} \underline{a}_{2} d_{2}}^{j}+R_{d_{1} b_{1} d_{2}}^{i} \tilde{R}_{\underline{a}_{2} b_{2} \underline{c}_{2}}^{j}\right)=0,
\end{aligned}
$$

where we again underlined antisymmetrised indices. By the antisymmetry of algebraic curvature tensors in the second index pair, all but the fourth and fifth term in the parenthesis vanish and we get

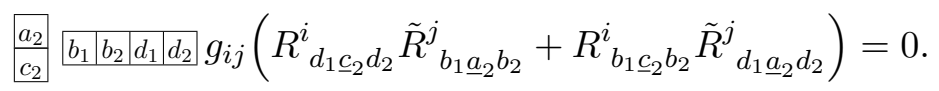

Due to the symmetrisation and antisymmetrisation we can permute the indices of the terms inside the parenthesis to find that

$$
\begin{gathered}
a_{2} \\
\hline c_{2}
\end{gathered} \underline{b_{1}\left|b_{2}\right| d_{1}\left|d_{2}\right|} g_{i j} R_{b_{1} \underline{a}_{2} b_{2}}^{i} \tilde{R}_{d_{1} \underline{c}_{2} d_{2}}^{j}=0 .
$$

Recall that this equation has been obtained from (5.4b) by a symmetrisation. This proves (5.4b) $\Rightarrow(5.4 \mathrm{c})$. The converse follows easily by antisymmetrising (5.4c) in $b_{1}, a_{2}, c_{2}$. This achieves the proof of the theorem.

Remark 5.4. Obviously (5.4b) must be true for $\tilde{R}=R$. This fact is not evident, but has been proven in [41].

For diagonal algebraic curvature tensors $R$ and $\tilde{R}$ on a four-dimensional vector space the condition $(5.4 \mathrm{c})$ can be written in the form

$$
\operatorname{det}\left(\begin{array}{ccc}
1 & R_{i j i j} & \tilde{R}_{i j i j} \\
1 & R_{j k j k} & \tilde{R}_{j k j k} \\
1 & R_{k i k i} & \tilde{R}_{k i k i}
\end{array}\right)=0
$$

for all distinct $i, j, k \in\{0,1,2,3\}$. 
Corollary 5.5. Stäckel lines ${ }^{5}$ in the KS-variety correspond to Stäckel systems. More precisely, the preimage of a Stäckel line under the map from integrable Killing tensors with diagonal algebraic curvature tensor to the KS-variety is a Stäckel system. In particular, the Killing tensors in a Stäckel system have simultaneously diagonalisable algebraic curvature tensors.

Proof. A Stäckel line is the projective line through the (collinear but not coinciding) points $M$, $\iota(\pi(M))$ and $\nu(\pi(M))$ in the KS-variety for any non-singular KS-matrix $M$. We have to prove that corresponding Killing tensors $K_{0}, K_{1}$ and $K_{2}$ mutually commute. We first show that $K_{1}$ and $K_{2}$ commute. Let $R$ and $\tilde{R}$ be their diagonal algebraic curvature tensors. From their KS-matrices (4.8) and $\Delta_{\alpha}=w_{\beta}-w_{\gamma}$ we can read off the values

$$
w_{\alpha}=0, \quad t_{\alpha}=n_{\alpha}, \quad \tilde{w}_{\alpha}=n_{\alpha}^{2}, \quad \tilde{t}_{\alpha}=n_{\beta} n_{\gamma},
$$

where we have neglected the scalar curvature, which can be chosen appropriately. The diagonals of $R$ and $\tilde{R}$ are then given by the columns of the matrix

$$
\left(\begin{array}{lll}
1 & R_{0101} & \tilde{R}_{0101} \\
1 & R_{0202} & \tilde{R}_{0202} \\
1 & R_{0303} & \tilde{R}_{0303} \\
1 & R_{2323} & \tilde{R}_{2323} \\
1 & R_{3131} & \tilde{R}_{3131} \\
1 & R_{1212} & \tilde{R}_{1212}
\end{array}\right)=\left(\begin{array}{ccc}
1 & w_{1}+t_{1} & \tilde{w}_{1}+\tilde{t}_{1} \\
1 & w_{2}+t_{2} & \tilde{w}_{2}+\tilde{t}_{2} \\
1 & w_{3}+t_{3} & \tilde{w}_{3}+\tilde{t}_{3} \\
1 & w_{1}-t_{1} & \tilde{w}_{1}-\tilde{t}_{1} \\
1 & w_{2}-t_{2} & \tilde{w}_{2}-\tilde{t}_{2} \\
1 & w_{3}-t_{3} & \tilde{w}_{3}-\tilde{t}_{3}
\end{array}\right)=\left(\begin{array}{ccc}
1 & +n_{1} & n_{1}^{2}+n_{2} n_{3} \\
1 & +n_{2} & n_{2}^{2}+n_{3} n_{1} \\
1 & +n_{3} & n_{3}^{2}+n_{1} n_{2} \\
1 & -n_{1} & n_{1}^{2}-n_{2} n_{3} \\
1 & -n_{2} & n_{2}^{2}-n_{3} n_{1} \\
1 & -n_{3} & n_{3}^{2}-n_{1} n_{2}
\end{array}\right) .
$$

The four square matrices in (5.11) can be obtained from this matrix by discarding the rows $(1,2,3),(1,5,6),(2,4,6)$ respectively $(3,4,5)$ and it is not difficult to see that their determinants vanish. This shows that $K_{1}$ and $K_{2}$ commute.

If $\iota(\pi(M))$ and $\nu(\pi(M))$ are linearly independent, then $M$ is a linear combination of both and hence $K_{0}$ is a linear combination of $g, K_{1}$ and $K_{2}$. In other words, $K_{0}, K_{1}$ and $K_{2}$ mutually commute.

If $\iota(\pi(M))$ and $\nu(\pi(M))$ are linearly dependent, then $n=\pi(M)$ satisfies $\left|n_{1}\right|=\left|n_{2}\right|=\left|n_{3}\right|$. For simplicity, suppose $n_{1}=n_{2}=n_{3}=1$. The other choices of the signs are analogous. By Lemma 4.7, $M$ is of the form (4.6a) and thus has $w_{\alpha}=-t_{\alpha}$. Consequently, the diagonal algebraic curvature tensors $R$ and $\tilde{R}$ of $K_{0}$ respectively $K_{2}$ are given by

$$
\left(\begin{array}{ccc}
1 & R_{0101} & \tilde{R}_{0101} \\
1 & R_{0202} & \tilde{R}_{0202} \\
1 & R_{0303} & \tilde{R}_{0303} \\
1 & R_{2323} & \tilde{R}_{2323} \\
1 & R_{3131} & \tilde{R}_{3131} \\
1 & R_{1212} & \tilde{R}_{1212}
\end{array}\right)=\left(\begin{array}{ccc}
1 & w_{1}+t_{1} & \tilde{w}_{1}+\tilde{t}_{1} \\
1 & w_{2}+t_{2} & \tilde{w}_{2}+\tilde{t}_{2} \\
1 & w_{3}+t_{3} & \tilde{w}_{3}+\tilde{t}_{3} \\
1 & w_{1}-t_{1} & \tilde{w}_{1}-\tilde{t}_{1} \\
1 & w_{2}-t_{2} & \tilde{w}_{2}-\tilde{t}_{2} \\
1 & w_{3}-t_{3} & \tilde{w}_{3}-\tilde{t}_{3}
\end{array}\right)=\left(\begin{array}{ccc}
1 & 0 & 2 \\
1 & 0 & 2 \\
1 & 0 & 2 \\
1 & 2 t_{1} & 0 \\
1 & 2 t_{2} & 0 \\
1 & 2 t_{3} & 0
\end{array}\right) .
$$

As above, they satisfy the commutation condition (5.11) and we conclude that $K_{0}, K_{1}$ and $K_{2}$ commute.

It remains to prove that every Stäckel system consists of Killing tensors with mutually diagonalisable algebraic curvature tensors. This follows from the fact that every Stäckel system contains a Killing tensor with simple eigenvalues and that such a Killing tensor $K$ uniquely determines the Stäckel system [3]. By the very definition of a Stäckel system, $K$ is integrable and by Theorem 1.13 we can assume it to have a diagonal algebraic curvature tensor. The corresponding point on the KS variety lies on a Stäckel line. By the above, $K$ then lies in a Stäckel system with diagonal algebraic curvature tensors. This proves the statement, since the Stäckel system determined by $K$ is unique.

\footnotetext{
${ }^{5}$ Recall Definition 4.10.
} 
Remark 5.6. Our result also shows that every integrable Killing tensor on $S^{3}$ is contained in a Stäckel system, not only those with simple eigenvalues. This follows from the above corollary in conjunction with Proposition 4.8.

We can now prove Theorem 1.19. Recall from Proposition 4.8 that each Stäckel line contains a unique skew symmetric point $\iota(n)$, where $n \in \mathbb{P}^{2}$, and that each non-singular skew symmetric point $\iota(n)$ determines a unique Stäckel line, given by the two points $\iota(n) \neq \nu(n)$. Moreover, the singular skew symmetric points are the four points $n=( \pm 1: \pm 1: \pm 1) \in \mathbb{P}^{2}$ for which $\iota(n)=\nu(n)$. Hence it suffices to show that the subspace spanned by $\iota(n(t))$ and $\nu(n(t))$ has a well defined limit for $t \rightarrow 0$ if $n(0)$ is one of these and that this limit depends on $\dot{n}(0) \in \mathbb{P}^{2}$. We leave it to the reader to verify that the limit space is spanned by $\iota(n(0))$ and $\iota(\dot{n}(0))$.

\section{Geometric constructions of integrable Killing tensors}

In this section we present several geometric constructions of integrable Killing tensors and interpret each of them within our algebraic picture from the last section.

\subsection{Special Killing tensors}

There are two ways in which integrable Killing tensors are related to geodesically equivalent metrics. The first is via special conformal Killing tensors.

Definition 6.1. A special conformal Killing tensor on a Riemannian manifold is a symmetric tensor $L_{\alpha \beta}$ satisfying the Sinjukov equation

$$
\nabla_{\gamma} L_{\alpha \beta}=\lambda_{\alpha} g_{\beta \gamma}+\lambda_{\beta} g_{\alpha \gamma},
$$

where

$$
\lambda=\frac{1}{2} \nabla \operatorname{tr} L
$$

as can be seen from contracting $\alpha$ and $\beta$.

Special conformal Killing tensors parametrise geodesically equivalent metrics in the following way [44].

Theorem 6.2. A metric $\tilde{g}$ is geodesically equivalent to $g$ if and only if the tensor

$$
L:=\left(\frac{\operatorname{det} \tilde{g}}{\operatorname{det} g}\right)^{\frac{1}{n+1}} \tilde{g}^{-1}
$$

is a special conformal Killing tensor.

From the definition we see that special conformal Killing tensors on a manifold $M$ form a vector space which is invariant under the isometry group of $M$. In other words, they define a representation of this group. The following lemma shows that this representation is isomorphic to a subrepresentation of the representation of the isomorphism group on Killing tensors.

Lemma 6.3. If $L$ is a special conformal Killing tensor, then $K:=L-(\operatorname{tr} L) g$ is a Killing tensor. This defines an injective map from the space of special conformal Killing tensors to the space of Killing tensors, which is equivariant with respect to the action of the isometry group. 
Proof. It is straightforward to check the first statement. Taking the trace on both sides of $K=L-(\operatorname{tr} L) g$ yields

$$
\operatorname{tr} K=(1-n) \operatorname{tr} L
$$

and hence

$$
L=K-\frac{\operatorname{tr} K}{n-1} g .
$$

This shows injectivity. Equivariance is obvious.

Definition 6.4. We define a special Killing tensor to be a Killing tensor of the form

$$
K=L-(\operatorname{tr} L) g,
$$

where $L$ is a special conformal Killing tensor.

Proposition 6.5. A special conformal Killing tensor as well as the corresponding special Killing tensor have vanishing Nijenhuis torsion. In particular, special Killing tensors are integrable.

Proof. Substituting the Sinjukov equation (6.1) into the expression (1.3) for $K=L$ shows that the Nijenhuis torsion of $L$ is zero. Substituting $K=L-(\operatorname{tr} L) g$ into (1.3) shows that this implies that the Nijenhuis torsion of $K$ is also zero.

For a non-flat constant curvature manifold $M \subset V$ we can identify the subrepresentation of special Killing tensors inside the space of Killing tensors. Recall that the space of Killing tensors is isomorphic to the space of algebraic curvature tensors on $V$ and an irreducible $\mathrm{GL}(V)$ representation. Under the subgroup $\mathrm{SO}(V) \subset \mathrm{GL}(V)$ this space decomposes into a Weyl component and a Ricci component.

Definition 6.6. The Kulkarni-Nomizu product of two symmetric tensors $h$ and $k$ on a vector space $V$ is the algebraic curvature tensor $h \otimes k$, given by

$$
(h \otimes k)_{a_{1} b_{1} a_{2} b_{2}}:=h_{a_{1} a_{2}} k_{b_{1} b_{2}}-h_{a_{1} b_{2}} k_{b_{1} a_{2}}-h_{b_{1} a_{2}} k_{a_{1} b_{2}}+h_{b_{1} b_{2}} k_{a_{1} a_{2}} .
$$

The Ricci component consists of all algebraic curvature tensors of the form $h \otimes g$, where $h$ is a symmetric tensor. Therefore the dimension of the Ricci part is equal to $N(N+1) / 2$ where $N=\operatorname{dim} V$. This is exactly the dimension of the space of special conformal Killing tensors on $M$ [44]. But there is no other $\mathrm{SO}(V)$-subrepresentation of the same dimension inside the space of algebraic curvature tensors. This shows the following.

Lemma 6.7. A special Killing tensor on a non-flat constant curvature manifold is a Killing tensor whose algebraic curvature tensor $R$ has a vanishing Weyl tensor. That is, $R$ is of the form

$$
R=h \otimes g
$$

for some symmetric tensor $h$ on $V$, where “ $₫$ " denotes the Kulkarni-Nomizu product (6.4).

Alternatively, this lemma can be checked directly by verifying that (6.3) satisfies (6.1) if $K$ has an algebraic curvature tensor of the form $R=h \otimes g$.

The following consequence is a special case of the so called "cone construction" [32]: Any special conformal Killing tensor on a Riemannian manifold $M$ can be extended to a covariantly constant symmetric tensor on the metric cone over $M$. In our case, where $M \subset V$ is a constant curvature manifold, this cone is nothing but the embedding space $V$. That is why a special conformal Killing tensor is the restriction of a constant symmetric tensor on the ambient space. 
Proposition 6.8. Let $R=h \otimes g$ be the algebraic curvature tensor of a special Killing tensor $K=L-(\operatorname{tr} L) g$ on a non-flat constant curvature manifold $M \subset V$. Then the corresponding special conformal Killing tensor

$$
L=K-\frac{\operatorname{tr} K}{n-1} g
$$

is the restriction of the (constant) symmetric tensor

$$
\hat{L}=h-\frac{\operatorname{Tr} h}{n-1} g
$$

from $V$ to $M$, where "Tr" denotes the trace on $V$ and "tr" the trace on the tangent space. In particular, if $h$ is tracefree, then $L$ is simply the restriction of $h$ from $V$ to $M$.

Proof. The special Killing tensor $K$ is given by substituting $R=h \otimes g$ into (1.6) using (6.4). This yields

$$
K_{x}(v, w)=h(x, x) g(v, w)-h(x, v) g(x, w)-h(x, w) g(x, v)+h(v, w) g(x, x)
$$

for tangent vectors $v, w \in T_{x} M$ at a point $x \in M$, i.e. vectors $x, v, w \in V$ with $g(x, x)=1$ and $g(x, v)=g(x, w)=0$. This simplifies to

$$
K_{x}(v, w)=h(v, w)+h(x, x) g(v, w) .
$$

Let $e_{1}, \ldots, e_{n}$ be an orthonormal basis of $T_{x} M$ and complete it with $e_{0}:=x$ to an orthonormal basis of $V$. Then

$$
\begin{aligned}
\operatorname{tr} K & =\sum_{\alpha=1}^{n} K_{x}\left(e_{\alpha}, e_{\alpha}\right)=\sum_{\alpha=1}^{n}\left(h\left(e_{\alpha}, e_{\alpha}\right)+h\left(e_{0}, e_{0}\right) g\left(e_{\alpha}, e_{\alpha}\right)\right) \\
& =\sum_{i=0}^{n} h\left(e_{i}, e_{i}\right)+(n-1) h\left(e_{0}, e_{0}\right)=\operatorname{Tr} h+(n-1) h(x, x) .
\end{aligned}
$$

Recall that "tr" denotes the trace on $T_{x} M$, whereas "Tr" denotes the trace on $V$. Substituting (6.7) and (6.8) into the right hand side of (6.5) now yields (6.6).

For $S^{3}$ we can verify the integrability of a special Killing tensor on $S^{3}$ algebraically, using the Theorems 1.13 and 1.14:

\section{Lemma 6.9.}

1. $R=h \otimes g$ is diagonalisable. More precisely, $R$ is diagonal in a basis where $h$ is diagonal. In this basis the diagonal elements of $R$ are given in terms of the diagonal elements $h_{i}:=h_{i i}$ by

$$
R_{i j i j}=h_{i}+h_{j}, \quad i \neq j .
$$

2. If $\operatorname{dim} V=4$, the $K S$-matrix (1.9) of $R=h \otimes g$ is antisymmetric with

$$
\Delta_{\alpha}=0, \quad t_{\alpha}=h_{0}+h_{\alpha} .
$$

In particular it has determinant zero.

Corollary 6.10. The space of special Killing tensors on $S^{3}$ corresponds to the projective space of antisymmetric matrices inside the KS-variety.

Proposition 4.8 and Corollary 5.5 now give a unique representative in each Stäckel system.

Corollary 6.11. Every Stäckel system on $S^{3}$ contains a special Killing tensor which is unique up to multiplication with constants and addition of multiples of the metric. 


\subsection{Killing tensors of Benenti type}

The second way in which integrable Killing tensors arise from geodesically equivalent metrics is described by the following theorem [2, 30, 33, 39].

Theorem 6.12. If a metric $\tilde{g}$ on a (pseudo-)Riemannian manifold is geodesically equivalent to $g$, then

$$
K:=\left(\frac{\operatorname{det} g}{\operatorname{det} \tilde{g}}\right)^{\frac{2}{n+1}} \tilde{g}
$$

is an integrable Killing tensor for $g$.

Definition 6.13. We will call a Killing tensor of the form (6.9) a Benenti-Killing tensor.

Recall that the standard metric on the unit sphere $S^{n} \subset V$ in a Euclidean vector space $(V, g)$ is the restriction of the scalar product $g$ from $V$ to $S^{n}$, which we denoted by $g$ as well. Its geodesics are the great circles on $S^{n}$. Consider the map

$$
\begin{aligned}
f: \quad S^{n} & \rightarrow S^{n}, \\
x & \mapsto f(x):=\frac{A x}{\|A x\|},
\end{aligned}
$$

for $A \in \mathrm{GL}(V)$. Since $A$ takes hyperplanes to hyperplanes, $f$ takes great circles to great circles. Hence the pullback $\tilde{g}$ of $g$ under $f$ is geodesically equivalent to $g$ by definition. The Killing tensor obtained from applying (6.9) to $\tilde{g}$ is given by [41]

$$
K_{x}(v, w)=\frac{g(A x, A x) g(A v, A w)-g(A x, A v) g(A x, A w)}{(\operatorname{det} A)^{\frac{4}{n+1}}}, \quad v, w \in T_{x} M .
$$

Comparing with (1.6), we see that the algebraic curvature tensor of this Killing tensor is proportional to $h \otimes h$, where " $₫$ " denotes the Kulkarni-Nomizu product (6.4) and

$$
h(v, w):=g(A v, A w)=g\left(v, A^{t} A w\right) .
$$

Moreover, we can replace the scalar product $h$ by any - not necessarily positive definite symmetric tensor. This motivates the following definition.

Definition 6.14. We will say a Killing tensor on a constant curvature manifold of Benenti type if its algebraic curvature tensor is proportional to

$$
R=h \otimes h
$$

for some symmetric tensor $h$ on $V$.

A Killing tensor of Benenti type with positive definite $h$ is a Benenti-Killing tensor and thus integrable. With the aid of the algebraic integrability conditions one can verify that any Killing tensor of Benenti type is integrable [41]. On $S^{3}$ we can check this directly, using the Theorems 1.13 and 1.14:

\section{Lemma 6.15.}

1. For a symmetric tensor $h$ on a vector space $V$, the algebraic curvature tensor $R=h \otimes h$ is diagonalisable. More precisely, $R$ is diagonal in a basis where $h$ is diagonal. In this basis the diagonal elements of $R$ are given in terms of the diagonal elements $h_{i}:=h_{i i}$ by

$$
R_{i j i j}=2 h_{i} h_{j}, \quad i \neq j .
$$

2. If $\operatorname{dim} V=4$, the KS-matrix (1.9) of $R=h \otimes h$ is given by

$$
\Delta_{\alpha}=\left(h_{0}-h_{\alpha}\right)\left(h_{\beta}-h_{\gamma}\right), \quad t_{\alpha}=h_{0} h_{\alpha}-h_{\beta} h_{\gamma}
$$

and has zero determinant and trace. 


\subsection{Benenti systems}

Special Killing tensors and Benenti-Killing tensors only constitute particular cases of a more general construction of integrable Killing tensors out of special conformal Killing tensors. This construction appears in the literature in different guises and under a number of different names: as "Newtonian systems of quasi-Lagrangian type" [40], as "systems admitting special conformal Killing tensors" [8], as "cofactor systems" [31], as "bi-Hamiltonian structures" [6, 16], as "biquasi-Hamiltonian systems" [9, 10], as " $L$-systems" [4] or as "Benenti systems" [7]. Here we follow Bolsinov and Matveev, who call them "Benenti systems" [7], because their formulation is directly reflected in our algebraic description for $S^{3}$.

Recall that the adjugate matrix $\operatorname{Adj} M$ of a matrix $M$ is the transpose of its cofactor matrix, which is equal to $(\operatorname{det} M) M^{-1}$ if $M$ is invertible. Given a metric, we can identify endomorphisms and bilinear forms. This allows us to extend this definition to bilinear forms. As for endomorphisms, we denote the adjugate of a bilinear form $L$ by $\operatorname{Adj} L$, which is again a bilinear form.

Theorem 6.16 ([7]). For a special conformal Killing tensor $L$ the family

$$
K(\lambda)=\operatorname{Adj}(L-\lambda g), \quad \lambda \in \mathbb{R}
$$

is a family of mutually commuting integrable Killing tensors. In particular, for $\lambda=0$ we recover the Benenti-Killing tensor (6.9) from (6.2).

Definition 6.17. The family $K(\lambda)=\operatorname{Adj}(L-\lambda g)$ of mutually commuting Killing tensors is called the Benenti system of the special conformal Killing tensor $L$.

In order to state the algebraic counterpart of the above theorem for constant curvature manifolds, we first need the following lemma.

Lemma 6.18. Let $h$ be a symmetric tensor on a vector space $V$ of dimension $n+1$. Then the algebraic curvature tensor

$$
\frac{(\operatorname{Adj} h) \otimes(\operatorname{Adj} h)}{\operatorname{det} h}
$$

is well defined and homogeneous in $h$ of degree $n-1$.

Proof. Chose a basis in which $h$ is diagonal with diagonal elements $h_{i}:=h_{i i}$. Then Adj $h$ is also diagonal and has diagonal elements $h_{0} \cdots h_{i-1} h_{i+1} \cdots h_{n}$. By (6.10) the algebraic curvature tensor (6.11) is diagonal and given by

$$
\begin{aligned}
R_{i j i j} & =2 \frac{h_{0} \cdots h_{i-1} h_{i+1} \cdots h_{n} \cdot h_{0} \cdots h_{j-1} h_{j+1} \cdots h_{n}}{h_{0} \cdots h_{n}} \\
& =2 h_{0} \cdots h_{i-1} h_{i+1} \cdots h_{j-1} h_{j+1} \cdots h_{n}
\end{aligned}
$$

for $i \neq j$. This is well defined and homogeneous in $h$ of degree $n-1$.

Proposition 6.19 (algebraic representation of Benenti systems). Let $L$ be a special conformal Killing tensor on a non-flat constant curvature manifold $M \subset V$, i.e. the restriction of $a$ (constant) symmetric tensor $\hat{L}$ from $V$ to $M$. To avoid confusion, we denote the metric on $V$ by $\hat{g}$ and its restriction to $M \subset V$ by $g$.

Then the Killing tensors of the Benenti system

$$
K=\operatorname{Adj}(L-\lambda g), \quad \lambda \in \mathbb{R}
$$


correspond to the algebraic curvature tensors

$$
R=\frac{\operatorname{Adj}(\hat{L}-\lambda \hat{g}) \otimes \operatorname{Adj}(\hat{L}-\lambda \hat{g})}{\operatorname{det}(\hat{L}-\lambda \hat{g})} .
$$

In particular, a member of this Benenti system is of Benenti type unless $\lambda$ is an eigenvalue of $\hat{L}$ and it comes from a geodesically equivalent metric if $-\lambda$ is sufficiently large.

Proof. For simplicity of notation, we absorb the trace terms " $\lambda g$ " and " $\lambda \hat{g}$ " into $L$ respectively $\hat{L}$ by formally setting $\lambda$ to 0 . At a fixed point $x \in M$ we consider the block decomposition of the endomorphism $\hat{\mathbf{L}}$ under the splitting $V=T_{x} M \oplus \mathbb{R} x$. Using the blockwise LU decomposition of $\hat{\mathbf{L}}$,

$$
\hat{\mathbf{L}}=\left(\begin{array}{ll}
\mathbf{L} & l \\
l^{t} & n
\end{array}\right)=\left(\begin{array}{cc}
\mathbf{L} & 0 \\
l^{t} & 1
\end{array}\right)\left(\begin{array}{cc}
\mathbf{I} & \mathbf{L}^{-1} l \\
0 & n-l^{t} \mathbf{L}^{-1} l
\end{array}\right),
$$

we can compute the block decomposition of the adjugate of $\hat{\mathbf{L}}$ :

$$
\operatorname{Adj} \hat{\mathbf{L}}=\left(\begin{array}{cc}
\frac{1}{\operatorname{det} \mathbf{L}}\left(\operatorname{det} \hat{\mathbf{L}} \operatorname{Adj} \mathbf{L}+(\operatorname{Adj} \mathbf{L}) l l^{t}(\operatorname{Adj} \mathbf{L})^{t}\right) & -(\operatorname{Adj} \mathbf{L}) l \\
-l^{t}(\operatorname{Adj} \mathbf{L})^{t} & \operatorname{det} \mathbf{L}
\end{array}\right) .
$$

Note that we have assumed without loss of generality that $\mathbf{L}$ is invertible. Now take a basis $e_{1}, \ldots, e_{n}$ of $T_{x} M$ and complete it with $e_{0}:=x$ to a basis of $V$. With respect to this basis the components of the Killing tensor $K$ corresponding to the algebraic curvature tensor (6.13) read

$$
K_{i j}=R_{0 i 0 j}=\frac{(\operatorname{Adj} \hat{L})_{00}(\operatorname{Adj} \hat{L})_{i j}-(\operatorname{Adj} \hat{L})_{0 i}(\operatorname{Adj} \hat{L})_{0 j}}{\operatorname{det} \hat{L}}=(\operatorname{Adj} L)_{i j} .
$$

That is, $K$ is given by (6.12), as was to be shown.

The adjugate of an $(n \times n)$-matrix $M$ is a polynomial in $M$ of degree $n-1$, given by

$$
\operatorname{Adj} M=\left.\frac{\chi_{M}(\lambda)-\chi_{M}(0)}{\lambda}\right|_{\lambda=M},
$$

where $\chi_{M}$ is the characteristic polynomial of $M$. Therefore a Benenti system is a polynomial in $\lambda$ of degree $n-1$ :

$$
K(\lambda)=\operatorname{Adj}(L-\lambda g)=\sum_{j=0}^{n-1} K_{j} \lambda^{j}
$$

Up to signs, the leading coefficient of this polynomial is the metric, the next coefficient is the special Killing tensor (6.2) and the constant term is the Benenti-Killing tensor (6.9):

$$
\begin{aligned}
& K_{n-1}=\lim _{\lambda \rightarrow \infty} \frac{1}{\lambda^{n-1}} K(\lambda)=\lim _{\lambda \rightarrow \infty} \operatorname{Adj}\left(\frac{1}{\lambda} L-g\right)=(-1)^{n-1} g, \\
& K_{n-2}=\left.\frac{d}{d \lambda}\right|_{\lambda=0} \lambda^{n-1} K\left(\frac{1}{\lambda}\right)=\left.\frac{d}{d \lambda}\right|_{\lambda=0} \operatorname{Adj}(\lambda L-g)=(-1)^{n-1}(L-(\operatorname{tr} L) g), \\
& K_{0}=K(0)=\operatorname{Adj} L .
\end{aligned}
$$

Note that since all the $K(\lambda)$ commute, the $n$ coefficients $K_{j}$ span a vector space of mutually commuting integrable Killing tensors. If the $n$ coefficients are linearly independent, then this space is a Stäckel system and the $K_{j}$ form a basis therein. However, it is in general an open question under which conditions this will be the case. For $S^{3}$ we can derive the answer directly from our algebraic description. 
Lemma 6.20. For a symmetric tensor $h$ on a four dimensional vector space we have

$$
\frac{(\operatorname{Adj} h) \otimes(\operatorname{Adj} h)}{\operatorname{det} h}=*(h \otimes h) *,
$$

where $*$ is the Hodge star operator.

Remark 6.21. Regarding (2.2), the conjugation of an algebraic curvature tensor with the Hodge star is simply a sign change in the trace free Ricci part:

$$
\mathbf{R}=\mathbf{W}+\mathbf{T}+\mathbf{S}, \quad * \mathbf{R} *=\mathbf{W}-\mathbf{T}+\mathbf{S} .
$$

Proof. Choose a basis in which $h$ is diagonal with diagonal elements $h_{i}:=h_{i i}$. For simplicity of notation we assume that $\operatorname{det} h \neq 0$. Then $h_{i} \neq 0$ and $\operatorname{Adj} h$ is diagonal with diagonal elements $\operatorname{det} h / h_{i}$. Substituting Adj $h$ for $h$ in (6.10) and comparing to (6.10) shows that the diagonal algebraic curvature tensors

$$
\tilde{R}=(\operatorname{Adj} h) \otimes(\operatorname{Adj} h), \quad R=h \otimes h
$$

are related by

$$
\tilde{R}_{i j i j}=2 \frac{\operatorname{det} h}{h_{i}} \frac{\operatorname{det} h}{h_{j}}=2(\operatorname{det} h) \frac{h_{0} h_{1} h_{2} h_{3}}{h_{i} h_{j}}=(\operatorname{det} h) 2 h_{k} h_{l}=(\operatorname{det} h) R_{k l k l},
$$

for $\{i, j, k, l\}=\{0,1,2,3\}$. Now the identity (6.14) follows from (2.13) and the fact that $R$ and $* R *$ differ in the sign of the trace free Ricci part.

Proposition 6.22. Let $L$ be a special conformal Killing tensor on $S^{3} \subset V$, i.e. the restriction of a (constant) symmetric tensor $\hat{L}$ from $V$ to $S^{3}$, and consider the corresponding Benenti system

$$
K(\lambda)=\operatorname{Adj}(L-\lambda g)=K_{2} \lambda^{2}+K_{1} \lambda+K_{0} .
$$

Then the coefficients $K_{0}, K_{1}$ and $K_{2}$ span a Stäckel system if and only if $\hat{L}$ has no triple eigenvalue.

Proof. By Proposition 6.19 and the identity (6.14) the algebraic curvature tensor of $K(\lambda)$ is the polynomial

$$
R(\lambda)=*((\hat{L}-\lambda g) \otimes(\hat{L}-\lambda g)) *=\lambda^{2} g \otimes g+2 \lambda \hat{L} \otimes g+*(\hat{L} \otimes \hat{L}) *=: \lambda^{2} R_{2}+\lambda R_{1}+R_{0} .
$$

The coefficients $R_{0}, R_{1}$ and $R_{2}$ are the algebraic curvature tensors of $K_{0}, K_{1}$ and $K_{2}$ respectively. We can assume them to be diagonal. Since $K_{2}$ is minus the metric, $K_{0}, K_{1}$ and $K_{2}$ are linearly independent if and only if $R_{0}$ and $R_{1}$ define two distinct points in the KS-variety.

Choose a basis in which $\hat{L}$ is diagonal with the eigenvalues $\Lambda_{i}$ on the diagonal. Without loss of generality we can assume that $\hat{L}$ is trace free. Motivated by (2.12), we can thus parametrise $\hat{L}$ by a vector $n \in \mathbb{R}^{3}$ as

$$
\Lambda_{0}=\frac{n_{\alpha}+n_{\beta}+n_{\gamma}}{2}, \quad \Lambda_{\alpha}=\frac{n_{\alpha}-n_{\beta}-n_{\gamma}}{2},
$$

where

$$
n_{\alpha}=\Lambda_{0}+\Lambda_{\alpha}=-\left(\Lambda_{\beta}+\Lambda_{\gamma}\right)
$$

Then $\hat{L}$ has a triple eigenvalue if and only if $\left|n_{1}\right|=\left|n_{2}\right|=\left|n_{3}\right|$. This condition is equivalent to the condition that the KS-matrices $\nu(n)$ and $\iota(n)$ in (4.8) are proportional, i.e. define the same 
point in the KS-variety. Our proof will therefore be finished if we show that $R_{0}$ and $R_{1}$ define the points $\nu(n)$ respectively $\iota(n)$ in the KS-variety.

That the algebraic curvature tensor $R_{1}=2 \hat{L} \otimes g$ defines the point $\iota(n)$ is the statement of Lemma 6.9. And since $R_{0}=*(\hat{L} \otimes \hat{L}) *$ differs from $\hat{L} \otimes \hat{L}$ in the sign of the trace free Ricci part, Lemma 6.15 implies that for $R_{0}$ the KS-matrix (1.9) is given by

$$
\Delta_{\alpha}=\left(\Lambda_{0}-\Lambda_{\alpha}\right)\left(\Lambda_{\beta}-\Lambda_{\gamma}\right)=n_{\beta}^{2}-n_{\gamma}^{2}, \quad t_{\alpha}=-\left(\Lambda_{0} \Lambda_{\alpha}-\Lambda_{\beta} \Lambda_{\gamma}\right)=n_{\beta} n_{\gamma} .
$$

Now compare this to $\nu(n)$ in (4.8) to finish the proof.

The Benenti system of a special conformal Killing tensors $L$ defines a map $\mathbb{R} \rightarrow \mathcal{K}\left(S^{3}\right)$ given by $\lambda \mapsto K(\lambda)=\operatorname{Adj}(L-\lambda g)$. In the proof above we have actually shown that this defines a map from $\mathbb{P}^{1}=\mathbb{R} \cup\{\infty\}$ to the KS-variety which maps $0 \mapsto \nu(n)$ and $\infty \mapsto \iota(n)$, where $n \in \mathbb{P}^{2}$ is determined by $L$. If the points $\iota(n)$ and $\nu(n)$ coincide, the image of this map is a single point. If they are distinct, the image is - by definition - the Stäckel line through both points. In this case the image of the Benenti system is this Stäckel line without the point $\iota(n)$.

We can now prove Theorem 1.17. It suffices to show that the image of diagonal algebraic curvature tensors of the form $\lambda_{2} h \otimes h$ is dense in the KS-variety. By Proposition 6.19 the algebraic curvature tensors of this form are dense in a Benenti system, so it suffices to show that the image of Benenti systems in the KS-variety is dense. But this follows from the preceding paragraph and part 2 of Proposition 4.8.

\subsection{Extension of integrable Killing tensors}

If we fix an orthogonal decomposition $\mathbb{R}^{n_{1}+n_{2}+2}=\mathbb{R}^{n_{1}+1} \oplus \mathbb{R}^{n_{2}+1}$, then two algebraic curvature tensors $R_{1}$ on $\mathbb{R}^{n_{1}+1}$ and $R_{2}$ on $\mathbb{R}^{n_{2}+1}$ define an algebraic curvature tensor $R_{1} \oplus R_{2}$ on $\mathbb{R}^{n_{1}+n_{2}+2}$. Correspondingly, two Killing tensors $K_{1}$ on $S^{n_{1}}$ and $K_{2}$ on $S^{n_{2}}$ define a Killing tensor $K_{1} \oplus K_{2}$ on $S^{n_{1}+n_{2}+1}$. In particular, we can extend any Killing tensor $K$ on $S^{n-1}$ by zero to a Killing tensor $K \oplus 0$ on $S^{n}$.

From the algebraic integrability conditions we see that $K_{1} \oplus K_{2}$ is integrable if $K_{1}$ and $K_{2}$ are both integrable. This direct sum operation defines an embedding

$$
\mathcal{K}\left(S^{n_{1}}\right) \times \mathcal{K}\left(S^{n_{2}}\right) \hookrightarrow \mathcal{K}\left(S^{n_{1}+n_{2}+1}\right),
$$

where $\mathcal{K}\left(S^{n}\right)$ denotes the space of integrable Killing tensors on $S^{n}$. In particular we have an embedding

$$
\mathcal{K}\left(S^{n-1}\right) \hookrightarrow \mathcal{K}\left(S^{n}\right) .
$$

Definition 6.23. We will call (integrable) Killing tensors arising in the way described above extensions of Killing tensors from $\left(S^{n_{1}}, S^{n_{2}}\right)$ respectively $S^{n-1}$.

As an example, let us determine which integrable Killing tensors on $S^{3}$ are extensions from $S^{2}$ respectively $\left(S^{1}, S^{1}\right)$. Note that in dimension one and two all Killing tensors are integrable. This follows directly from the integrability conditions.

An algebraic curvature tensor $R$ on $\mathbb{R}^{4}$ is an extension by zero of an algebraic curvature tensor on $\mathbb{R}^{3}$ under the inclusion $\left(x_{1}, x_{2}, x_{3}\right) \mapsto\left(0, x_{1}, x_{2}, x_{3}\right)$ if and only if $R_{a_{1} b_{1} a_{2} b_{2}}$ is zero whenever one of the indices is zero. In particular, the diagonal algebraic curvature tensor (1.8) on $S^{3}$ is an extension from $S^{2}$ if and only if $w_{\alpha}+\frac{s}{12}=-t_{\alpha}$. In this case the KS-matrix (1.9) is equal to (4.6a) with $n_{1}=n_{2}=n_{3}$. Therefore the extensions of Killing tensors from $S^{2}$ correspond to the isokernel planes in the KS-variety. In particular, the face centers of the octahedron in the KS-variety correspond to the extension of the metric on $S^{2}$ under the four embeddings $S^{2} \subset S^{3}$ given by intersecting $S^{3} \subset \mathbb{R}^{4}$ with each of the four coordinate hyperplanes. 
An algebraic curvature tensor $R$ on $\mathbb{R}^{4}$ is a sum under the decomposition of $\left(x_{0}, x_{1}, x_{2}, x_{3}\right)$ into $\left(x_{0}, x_{1}\right)$ and $\left(x_{2}, x_{3}\right)$ if and only if the components $R_{a_{1} b_{1} a_{2} b_{2}}$ are zero unless all four indices are either in $\{0,1\}$ or in $\{2,3\}$. In particular, a diagonal algebraic curvature tensor (1.8) is an extension from $\left(S^{1}, S^{1}\right)$ if and only if $w_{2}+\frac{s}{12}=t_{2}=w_{3}+\frac{s}{12}=t_{3}=0$. In this case the KS-matrix (1.9) is equal to

$$
\left(\begin{array}{ccc}
0 & 0 & 0 \\
0 & -w_{1} & -t_{1} \\
0 & +t_{1} & +w_{1}
\end{array}\right)=-\frac{w_{1}-t_{1}}{2} V_{-1}-\frac{w_{1}+t_{1}}{2} V_{+1},
$$

where $V_{+1}$ and $V_{-1}$ are rank one singular points in the KS-variety, cf. (4.4). Therefore the extensions of Killing tensors from $\left(S^{1}, S^{1}\right)$ correspond to the projective lines joining opposite vertices of the octahedron in the KS-variety. In particular, each vertex of the octahedron corresponds to an extension of the metrics on two orthogonal copies of $S^{1}$ in $S^{3}$, given by intersecting $S^{3} \subset \mathbb{R}^{4}$ with a pair of orthogonal coordinate planes.

\section{Separation coordinates}

We eventually demonstrate how separation coordinates on $S^{3}$ and their classification arise naturally and in a purely algebraic way from our description of integrable Killing tensors.

Kalnins and Miller proved that separation coordinates on $S^{n}$ are always orthogonal [22]. From the work of Eisenhart we know that orthogonal separation coordinates are in bijective correspondence with Stäckel systems [13]. And in the preceding sections we have proven that on $S^{3}$ every Stäckel system contains a special Killing tensor which is essentially unique. By definition, special Killing tensors are in bijective correspondence with special conformal Killing tensors. But Crampin has shown that the eigenvalues of a special conformal Killing tensor are constant on the coordinate hypersurfaces of the corresponding separation coordinates [8]. Hence, if these eigenvalues are simple and non-constant, they can be used as separation coordinates. This reduces the classification of separation coordinates on $S^{3}$ to a computation of the eigenvalues of special conformal Killing tensors. The computation is considerably simplified by the fact that a special conformal Killing tensor on $S^{n} \subset V$ is the restriction of a (constant) symmetric tensor on $V$.

\subsection{Eigenvalues of special conformal Killing tensors}

Let $L$ be a special conformal Killing tensor on a non-flat constant curvature manifold $M \subset V$, i.e. the restriction from $V$ to $M$ of a (constant) symmetric tensor $\hat{L}$. From now on we consider $L$ and $\hat{L}$ as endomorphisms. We want to compute the eigenvalues $\lambda_{1}(x) \leqslant \cdots \leqslant \lambda_{n}(x)$ of $L: T_{x} M \rightarrow T_{x} M$ for a fixed point $x \in M$ and relate them to the (constant) eigenvalues $\Lambda_{0} \leqslant$ $\cdots \leqslant \Lambda_{n}$ of $\hat{L}: V \rightarrow V$.

Let $P: V \rightarrow V$ be the orthogonal projection from $V \cong T_{x} M \oplus \mathbb{R} x$ to $T_{x} M$. Then the restriction of $P \hat{L} P$ to $T_{x} M$ is $L$ and the restriction of $P \hat{L} P$ to $x$ is zero. Therefore $x$ is an eigenvector of $P \hat{L} P$ with eigenvalue 0 and the remaining eigenvectors and eigenvalues are those of $L$. This means the eigenvalue equation $L v=\lambda v$ is equivalent to $P \hat{L} P v=\lambda v$ for $v \perp x$. By the definition of $P$ we have $P v=v$ and $P \hat{L} v=\hat{L} v-g(\hat{L} v, x) x$. Hence we seek common solutions to the two equations

$$
(\hat{L}-\lambda) v=g(\hat{L} v, x) x, \quad g(x, v)=0 .
$$

If $\lambda$ is not an eigenvalue of $\hat{L}$, then $(\hat{L}-\lambda)$ is invertible and $v$ is proportional to $(\hat{L}-\lambda)^{-1} x$. The condition $v \perp x$ then yields the equation

$$
g\left((\hat{L}-\lambda)^{-1} x, x\right)=0
$$


for $\lambda$. In an eigenbasis of $\hat{L}$ this equation reads

$$
q(\lambda):=\sum_{k=0}^{n} \frac{x_{k}^{2}}{\Lambda_{k}-\lambda}=0 .
$$

That is, the zeroes of the function $q(\lambda)$ are eigenvalues of $L$. As depicted in Fig. 2, $q(\lambda)$ goes to 0 for $\lambda \rightarrow \pm \infty$, has poles at the eigenvalues $\Lambda_{k}$ of $\hat{L}$ unless $x_{k}=0$ and is monotonely increasing in between.

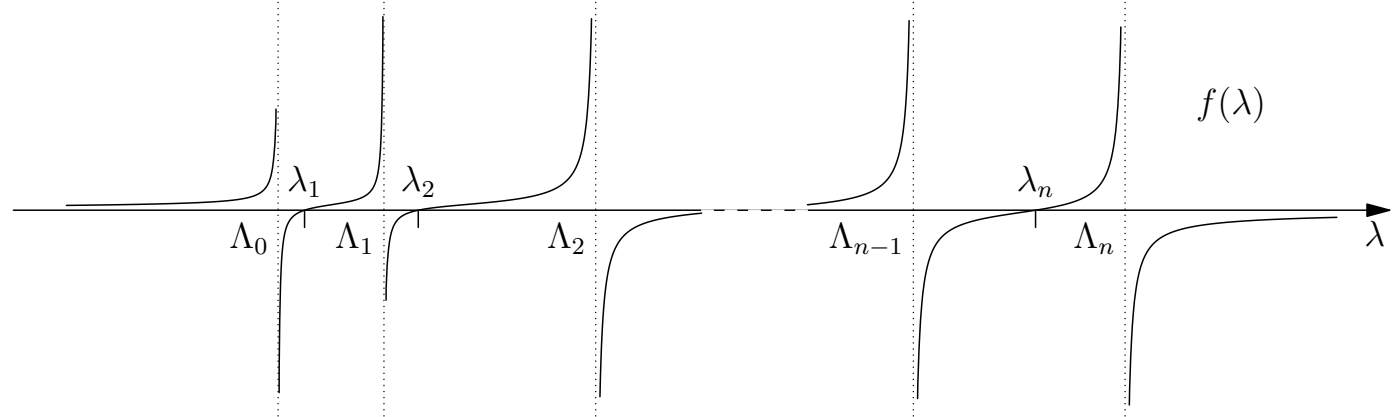

Figure 2. Eigenvalues of a special conformal Killing tensor on $S^{n}$.

Let us first suppose that $\hat{L}$ has only simple eigenvalues. In this case (7.1) is the defining equation for elliptic coordinates $\lambda_{1}(x), \ldots, \lambda_{n}(x)$ on $S^{n}$ with parameters $\Lambda_{0}<\Lambda_{1}<\cdots<$ $\Lambda_{n}$ [36]. If $x_{k} \neq 0$ for all $k=0, \ldots, n$, then $q$ has exactly $n+1$ poles at $\Lambda_{0}, \ldots, \Lambda_{n}$ and $n$ zeroes in between. If $x_{k}=0$, then $q$ has no pole at $\Lambda_{k}$. But $x_{k}=0$ means that the $k$-th eigenvector of $\hat{L}$ is orthogonal to $x$ and hence also an eigenvector of $L$ with eigenvalue $\Lambda_{k}$. We conclude that the eigenvectors $v_{k}$ of $\hat{L}$ which are orthogonal to $x$ are eigenvectors of $L$ with the same eigenvalue $\Lambda_{k}$, and that the remaining eigenvalues of $L$ are the zeroes $\lambda_{k}$ of the function $q(\lambda)$ with corresponding eigenvector $v_{k}=\left(\hat{L}-\lambda_{k}\right)^{-1} x$. In particular, the eigenvalues of $\hat{L}$ and $L$ intertwine:

$$
\Lambda_{0} \leqslant \lambda_{1}(x) \leqslant \Lambda_{1} \leqslant \lambda_{2}(x) \leqslant \cdots \leqslant \lambda_{n}(x) \leqslant \Lambda_{n} .
$$

Note that the common denominator of the fractions in the sum (7.1) is the characteristic polynomial $\chi_{\hat{L}}(\lambda)$ of $\hat{L}$. Therefore the function $q$ is the quotient of the characteristic polynomials of $L$ and $\hat{L}$.

If $\hat{L}$ has a simple eigenvalue, then the corresponding eigenspace is one dimensional and has only a trivial intersection with a generic tangent space to $M$, because $M \subset V$ is of codimension one. If $\hat{L}$ has a double eigenvalue, then the corresponding eigenspace is a plane and has a one-dimensional intersection with a generic tangent space to $M$. This defines a field of common eigenvectors for $L$ and $\hat{L}$ on $M$. The corresponding eigenvalue is the same for $L$ and $\hat{L}$ and hence constant on $M$. This also follows from (7.2). In the same way an eigenvalue of $\hat{L}$ with multiplicity $m+1$ defines an $m$-dimensional eigenspace distribution of $L$ with constant eigenvalue.

Proposition 7.1. Let $L$ be a special conformal Killing tensor on a constant curvature manifold $M \subset V$, i.e. the restriction of a constant symmetric trace free tensor $\hat{L}$ on $V$. Then:

1. If $\hat{L}$ has only simple eigenvalues, then the eigenvalues of $L$ define elliptic coordinates on $M$ whose parameters are the eigenvalues of $\hat{L}$.

2. Any multiple eigenvalue of $\hat{L}$ is a constant eigenvalue for $L$.

3. $L$ has simple eigenvalues if and only if $\hat{L}$ has at most double eigenvalues. 


\subsection{Killing tensors and separation coordinates on $S^{2}$}

Before we turn to $S^{3}$, let us briefly discuss integrable Killing tensors and separation coordinates on $S^{2}$. The reason is that their extensions will appear on $S^{3}$. First note that in dimension two every Killing tensor is integrable. So we do not have to deal with the integrability conditions. Note also that algebraic curvature tensors on $\mathbb{R}^{3}$ have a zero Weyl part and are thus determined by their Ricci tensor $T$ alone. This means that Killing tensors on $S^{2} \subset \mathbb{R}^{3}$ correspond to symmetric tensors $T$ on $\mathbb{R}^{3}$, where the metric corresponds to the identity.

Stäckel systems on $S^{2}$ are of dimension two and contain the metric. That implies that each Stäckel system on $S^{2}$ corresponds to a plane of Ricci tensors spanned by the identity and some trace free symmetric tensor, the latter being unique up to multiples. Moreover, the isometry group acts by conjugation on the Ricci tensor. Accordingly, the classification of separation coordinates on $S^{2}$ modulo isometries is equivalent to the classification of trace free symmetric tensors $T$ on $\mathbb{R}^{3}$ under the orthogonal group $\mathrm{O}(3)$.

In particular, every Killing tensor on $S^{2}$ is special and we can make use of the results in the previous section. Depending on the multiplicities of the eigenvalues $t_{1} \leqslant t_{2} \leqslant t_{3}$ of the trace free Ricci tensor $T$ of a Killing tensor on $S^{2}$ we consider the following two cases.

Elliptic coordinates. If $t_{1}<t_{2}<t_{3}$, then the restriction $L$ of $T$ from $\mathbb{R}^{3}$ to $S^{2} \subset \mathbb{R}^{3}$ has two different eigenvalues, given by the zeros of its characteristic polynomial

$$
\chi_{L}(\lambda)=\left(t_{2}-\lambda\right)\left(t_{3}-\lambda\right) x_{1}^{2}+\left(t_{3}-\lambda\right)\left(t_{1}-\lambda\right) x_{2}^{2}+\left(t_{1}-\lambda\right)\left(t_{2}-\lambda\right) x_{3}^{2}=0 .
$$

Using that $x_{1}^{2}+x_{2}^{2}+x_{3}^{2}=1$ and $t_{1}+t_{2}+t_{3}=0$ and writing the endomorphisms $T$ and $\operatorname{Adj} T$ as forms, this quadratic equation reads

$$
\lambda^{2}+T(x, x) \lambda+(\operatorname{Adj} T)(x, x)=0 .
$$

The two solutions $\lambda_{1}(x)$ and $\lambda_{2}(x)$ are real and satisfy $t_{1}<\lambda_{1}(x)<t_{2}<\lambda_{2}(x)<t_{3}$. As mentioned above, they define elliptic coordinates on $S^{2}$ with parameters $t_{1}<t_{2}<t_{3}$.

Spherical coordinates. If $t_{1}<t_{2}=t_{3}$, the characteristic polynomial of $L$ reduces to

$$
\chi_{L}(\lambda)=\left(t_{2}-\lambda\right)\left[\left(t_{2}-\lambda\right) x_{1}^{2}+\left(t_{1}-\lambda\right)\left(x_{2}^{2}+x_{3}^{2}\right)\right]=0 .
$$

One of the eigenvalues only depends on $x_{1}$ and the other is constant:

$$
\lambda_{1}(x)=t_{2} x_{1}^{2}+t_{1}\left(1-x_{1}^{2}\right), \quad \lambda_{2}(x)=t_{2} .
$$

The curves of constant $\lambda_{1}(x)$ are circles of constant latitude and the meridians complete them to an orthogonal coordinate system - the spherical coordinates.

The case $t_{1}=t_{2}<t_{3}$ is analogue and yields spherical coordinates as well. Note that even so, both coordinate systems are not isometric. This is because they differ not only by an isometry but also by an exchange of the two coordinates.

We can use the action of the isometry group $\mathrm{O}(3)$ to diagonalise the Ricci tensor and thereby the algebraic curvature tensor. Hence the projective variety of integrable Killing tensors on $S^{2}$ with diagonal algebraic curvature tensor is isomorphic to the projective plane $\mathbb{P}^{2}$, where the point $(1: 1: 1) \in \mathbb{P}^{2}$ corresponds to the metric on $S^{2}$. Moreover, Stäckel systems on $S^{2}$ correspond to projective lines through this point, since they always contain the metric. The residual isometry group action on Killing tensors with diagonal algebraic curvature tensor is the natural action of $S_{3}$ on $\mathbb{P}^{2}$ by permutations.

\subsection{The classification}

We now recover the well known classification of separation coordinates on $S^{3}[13,22]$. In particular, this will explain the graphical procedure given in [22] in terms of the classification 
Table 1. Classification of separation coordinates on $S^{3}$.

\begin{tabular}{lllcc}
\hline type & coordinates & induced from & {$[13]$} & {$[22]$} \\
\hline$(0123)$ & elliptic & - & V & $(1)$ \\
$(01(23))$ & oblate Lamé rotational & - & I & $(2 \mathrm{a})$ \\
$(0(12) 3)$ & prolate Lamé rotational & - & I & $(2 \mathrm{~b})$ \\
$((01)(23))$ & cylindrical & $\left(S^{1}, S^{1}\right)$ & III & $(5)$ \\
$(0(123))$ & Lamé subgroup reduction & $S^{2}$ elliptic & IV & $(3)$ \\
$(0(1(23)))$ & spherical & $S^{2}$ spherical & II & $(4)$ \\
\hline
\end{tabular}

of trace free symmetric tensors under the orthogonal group. The results are summarised in Table 1.

As before, we will denote by $\Lambda_{0} \leqslant \Lambda_{1} \leqslant \Lambda_{2} \leqslant \Lambda_{3}$ the ordered eigenvalues of the symmetric tensor $\hat{L}$ associated to a Stäckel system and parametrised by a vector $\left(n_{1}, n_{2}, n_{3}\right) \in \mathbb{R}^{3}$ via (6.15a). Recall that we have the freedom to add a multiple of the metric to a Killing tensor or to multiply it with a constant. Both operations only affect the eigenvalues, not the eigenspaces, and therefore result in different parametrisations of the same coordinate system. Whereas Kalnins and Miller use this freedom to set $\Lambda_{0}=0$ and $\Lambda_{1}=1$, here we use it to assume that $\hat{L}$ is trace free and then to consider $\left(\Lambda_{0}: \Lambda_{1}: \Lambda_{2}: \Lambda_{3}\right)$ respectively $\left(n_{1}: n_{2}: n_{3}\right)$ as a point in $\mathbb{P}^{2}$.

In particular, changing the sign of $\hat{L}$ reverses the order of the eigenvalues. This leaves us with the following five different alternatives for the multiplicities of the ordered set of eigenvalues of $\hat{L}$. For simplicity we write " $i$ " for " $\Lambda_{i}$ " and denote multiple eigenvalues by parenthesising them:

$$
\text { (0123), } \quad(01(23)), \quad(0(12) 3), \quad((01)(23)), \quad(0(123)) .
$$

(0123) Elliptic coordinates. We have already seen that if the eigenvalues of $\hat{L}$ are simple, then the eigenvalues of $L$ are also simple and define elliptic coordinates on $S^{n}$ given implicitly by $(7.1)$.

(01(23)) Oblate Lamé rotational coordinates. If $\hat{L}$ has a double eigenvalue $\Lambda_{k-1}=\Lambda_{k}$, then the corresponding eigenvalue $\lambda_{k}$ of $L$ is constant and can not be used as a coordinate. Nevertheless, we can derive the missing coordinate from the fact that the coordinate hypersurfaces are orthogonal to the eigenspaces of $L$. Let $E$ be an eigenplane of $\hat{L}$ and denote by $E^{\perp}$ its orthogonal complement in $V$. Recall that the corresponding eigenspace of $L$ at a point $x \in S^{n}$ is given by the intersection of $E$ with the tangent space $T_{x} S^{n}$. Its orthogonal complement in $T_{x} S^{n}$ is the intersection of $T_{x} S^{n}$ with the hyperplane that contains both $x$ and $E^{\perp}$. The coordinate hypersurfaces are therefore the intersections of hyperplanes containing $E^{\perp}$ with $S^{n}$ and can be parametrised by the polar angle in $E$. Hence this angle can be taken as a coordinate.

By (7.2), $L$ has single eigenvalues given by (7.1) if and only if $\hat{L}$ has at most double eigenvalues and every double eigenvalue of $\hat{L}$ determines a constant eigenvalue for $L$. If we replace every constant eigenvalue by the polar angle in the corresponding eigenplane of $\hat{L}$, we obtain a complete coordinate system. Concretely, in the case $\Lambda_{0}<\Lambda_{1}<\Lambda_{2}=\Lambda_{3}$ the separation coordinates are defined by the solutions $\lambda_{1}(x)$ and $\lambda_{2}(x)$ of (7.1) together with the polar angle in the $\left(x_{2}, x_{3}\right)$-plane.

For $\Lambda_{0}<\Lambda_{1}<\Lambda_{2}=\Lambda_{3}$ we have $n_{1}<n_{2}=n_{3}$. The corresponding Stäckel line thus goes through the two points in the KS-variety given by the KS-matrices (4.8):

$$
\left(\begin{array}{ccc}
0 & -n_{2} & n_{2} \\
n_{2} & 0 & -n_{1} \\
-n_{2} & n_{1} & 0
\end{array}\right), \quad\left(\begin{array}{ccc}
0 & -n_{1} n_{2} & n_{1} n_{2} \\
n_{1} n_{2} & n_{2}^{2}-n_{1}^{2} & -n_{2}^{2} \\
-n_{1} n_{2} & n_{2}^{2} & n_{1}^{2}-n_{2}^{2}
\end{array}\right)
$$


Subtracting $n_{1}$ times the first matrix from the second, we see that the same Stäckel line is also determined by the two points

$$
\left(\begin{array}{ccc}
0 & -n_{2} & n_{2} \\
n_{2} & 0 & -n_{1} \\
-n_{2} & n_{1} & 0
\end{array}\right), \quad V_{-1}=\left(\begin{array}{ccc}
0 & 0 & 0 \\
0 & +1 & -1 \\
0 & +1 & -1
\end{array}\right)
$$

(0(12)3) Prolate Lamé rotational coordinates. The case $\Lambda_{0}<\Lambda_{1}=\Lambda_{2}<\Lambda_{3}$ is analogue and yields Lamé rotational coordinates which differ from the above by an isometry and an exchange of two of the coordinates.

((01)(23)) Cylindrical coordinates. In the case $\Lambda_{0}=\Lambda_{1}<\Lambda_{2}=\Lambda_{3}$ equation (7.1) has only one non-constant solution, namely $\lambda_{2}(x)$ :

$$
\lambda_{1}=\Lambda_{1} \leqslant \lambda_{2}(x)=\Lambda_{1}\left(x_{2}^{2}+x_{3}^{2}\right)+\Lambda_{3}\left(x_{0}^{2}+x_{1}^{2}\right) \leqslant \lambda_{3}=\Lambda_{3} .
$$

The separation coordinates are obtained by replacing the constant eigenvalues $\lambda_{1}$ and $\lambda_{3}$ by the polar angle in the $\left(x_{0}, x_{1}\right)$ respectively $\left(x_{2}, x_{3}\right)$-plane.

For $\Lambda_{0}=\Lambda_{1}<\Lambda_{2}=\Lambda_{3}$ we have $n_{1}<n_{2}=n_{3}=0$ and the corresponding Stäckel line goes through the two points in the KS-variety given by the KS-matrices (4.8):

$$
\left(\begin{array}{ccc}
0 & 0 & 0 \\
0 & 0 & -n_{1} \\
0 & +n_{1} & 0
\end{array}\right), \quad\left(\begin{array}{ccc}
0 & 0 & 0 \\
0 & -n_{1}^{2} & 0 \\
0 & 0 & n_{1}^{2}
\end{array}\right) .
$$

Equivalently, this Stäckel line goes through the two points

$$
V_{-1}=\left(\begin{array}{ccc}
0 & 0 & 0 \\
0 & +1 & -1 \\
0 & +1 & -1
\end{array}\right), \quad V_{+1}=\left(\begin{array}{ccc}
0 & 0 & 0 \\
0 & +1 & +1 \\
0 & -1 & -1
\end{array}\right)
$$

i.e. opposed vertices of the octahedron in the KS-variety. We have seen that this describes extensions from $\left(S^{1}, S^{1}\right)$ to $S^{3}$.

(0(123)) Coordinates extended from $S^{2} \subset S^{3}$. In the remaining case $\Lambda_{0}<\Lambda_{1}=\Lambda_{2}=\Lambda_{3}$, equation (7.1) has the solutions

$$
\lambda_{1}(x)=\Lambda_{1} x_{0}^{2}+\Lambda_{0}\left(x_{1}^{2}+x_{2}^{2}+x_{3}^{2}\right), \quad \lambda_{2}=\lambda_{3}=\Lambda_{1} .
$$

We can take the non-constant eigenvalue $\lambda_{1}(x)$ as one separation coordinate on $S^{3}$. However, since the other two eigenvalues of $L$ are equal, we can not recover all separation coordinates from the special conformal Killing tensor $L$ in this case. We will have to consider another Killing tensor in the Stäckel system instead - one with simple eigenvalues.

Before we do so, let us collect some facts from previous sections for the case $\Lambda_{0}<\Lambda_{1}=\Lambda_{2}=\Lambda_{3}$.

Proposition 7.2. Consider the following objects associated to a system of separation coordinates on $S^{3}$ :

- the Stäckel system,

- a special conformal Killing tensor $L$ whose eigenvalues are constant on the coordinate hypersurfaces,

- the Benenti system $K(\lambda)=\operatorname{Adj}(L-\lambda g)$ of $L$,

- the special Killing tensor $K=L-(\operatorname{tr} L) g$ in the Stäckel system,

- the Stäckel line in the KS-variety, 
- the trace free symmetric tensor $\hat{L}$ on $V$ which restricts to $L$,

- the vector $\vec{n}=\left(n_{1}, n_{2}, n_{3}\right) \in \mathbb{R}^{3}$ parametrising $\hat{L}$ via (6.15a).

Then the following statements are equivalent:

1. The Stäckel system consists of extensions of Killing tensors from $S^{2} \subset S^{3}$ (up to the addition of a multiple of the metric).

2. L has a multiple eigenvalue (which is constant).

3. $K(\lambda)$ does not span the Stäckel system.

4. $K$ corresponds to a singular skew symmetric point in the KS-variety.

5. The Stäckel line lies on an isokernel plane in the KS-variety.

6. $\hat{L}$ has a triple eigenvalue.

7. $\left|n_{1}\right|=\left|n_{2}\right|=\left|n_{3}\right|$.

Proof. The equivalence of 6 and 7 is a direct consequence of (6.15a). The equivalence to the statements 1-5 follows from Section 6.4, Proposition 7.1, Proposition 6.22, Proposition 4.4 and Lemma 4.7, respectively.

To make the extension of Killing tensors from $S^{2} \subset S^{3}$ more explicit, consider a Killing tensor on $S^{2}$ with algebraic curvature tensor $R$ and Ricci tensor $T$ and choose a basis in which $T$ is diagonal with diagonal elements $t_{1}, t_{2}$ and $t_{3}$. Then $R$ is also diagonal and, if $T$ is trace free, given by

$$
R_{1212}=t_{1}+t_{2}=-t_{3}, \quad R_{2323}=t_{2}+t_{3}=-t_{1}, \quad R_{3131}=t_{3}+t_{1}=-t_{2} .
$$

In Section 6.4 we saw that this Killing tensor extends to a Killing tensor on $S^{3}$ corrsponding to the point in the KS-variety that is defined by the KS-matrix

$$
V_{t}:=\left(\begin{array}{ccc}
t_{3}-t_{2} & -t_{3} & t_{2} \\
t_{3} & t_{1}-t_{3} & -t_{1} \\
-t_{2} & t_{1} & t_{2}-t_{1}
\end{array}\right)=t_{1} V_{+1}+t_{2} V_{+2}+t_{3} V_{+3} .
$$

If $T$ is not trace free, we obtain the same result. This shows that the eigenvalues of the Ricci tensor of a Killing tensor on $S^{2}$ induce barycentric coordinates on the isokernel planes in the KS-variety.

In Section 7.2 we have already computed the eigenvalues $\lambda_{2}(x)$ and $\lambda_{3}(x)$ of a Killing tensor on $S^{2}$, parametrised by the eigenvalues of its Ricci tensor $T$. We see that if one perturbs the special Killing tensor on $S^{3}$ within a Stäckel system, the constant double eigenvalue $\lambda_{2}=\lambda_{3}$ splits up into two simple eigenvalues $\lambda_{2}(x)$ and $\lambda_{3}(x)$, which come from separation coordinates on $S^{2}$ under an embedding $S^{2} \subset S^{3}$. On the other hand, the eigenvalue $\lambda_{1}(x)$ remains unchanged. Together, they define separation coordinates on $S^{3}$. We denote this by writing the multiplicities of $\hat{L}$ as $(0(123))$ respectively $(0(1(23)))$, depending on whether the multiplicities of the Ricci tensor $T$ are (123) or (1(23)). This yields the remaining two systems of separation coordinates:

$(\mathbf{0}(\mathbf{1}(\mathbf{2 3})))$ Spherical coordinates. These are extensions of spherical coordinates on $S^{2}$ and correspond to Stäckel lines through a face center and a vertex of the octahedron in the KS-variety.

(0(123)) Lamé subgroup reduction. These are extensions of elliptic coordinates on $S^{2}$ and correspond to Stäckel lines through a face center, but not a vertex of the octahedron in the KS-variety. 


\subsection{The classification space}

The non-generic separation coordinates are characterised by $n_{1}= \pm n_{2}, n_{2}= \pm n_{3}$ and $n_{3}= \pm n_{1}$. This defines six projective lines that divide the projective plane of skew symmetric matrices in the KS-variety into twelve congruent triangles. The four singular skew symmetric points are given by $\left|n_{1}\right|=\left|n_{2}\right|=\left|n_{3}\right|$ and each of the triangles has two of them as vertices. Upon a blow-up in these points, the triangles become pentagons. Any of these pentagons constitutes a fundamental domain for the $S_{4}$-action. Regarding our notation for the multiplicities of $\hat{L}$, we realise that the vertices are labeled by the different possibilities to parenthesise the ordered set of eigenvalues of $\hat{L}$ with pairs of correctly matching parentheses and that the edges correspond to a single application of the associativity rule. This naturally identifies the classification space for separation coordinates on $S^{3}$ with the associahedron $K_{4}$, also known as Stasheff polytope [46].

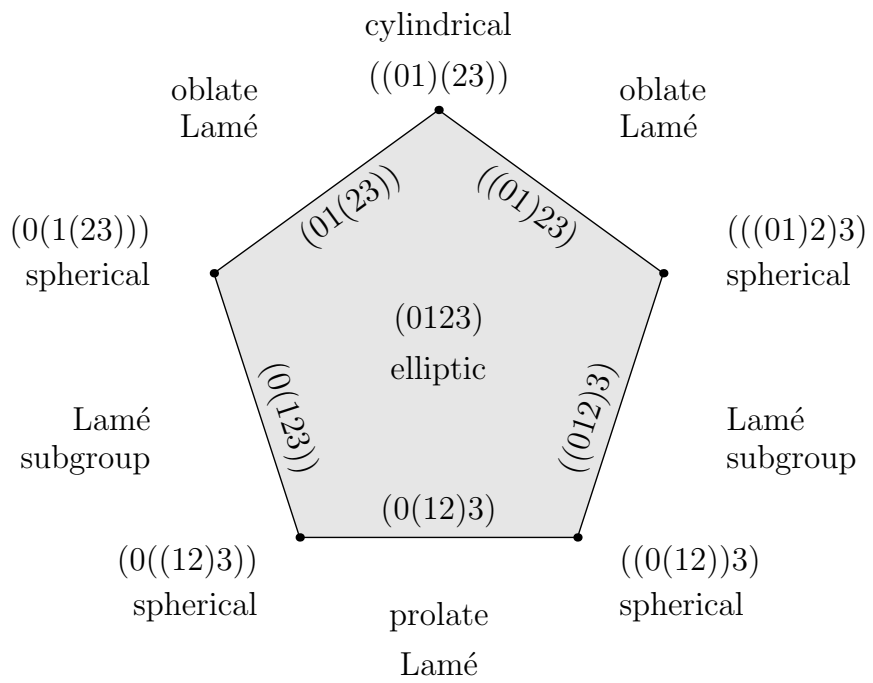

Figure 3. Identification of the moduli space of separation coordinates on $S^{3}$ with the associahedron $K_{4}$.

\section{Acknowledgements}

I would like to express my gratitude to Robert Milson for his motivation and the inspiring discussions about my findings. I would also like to thank Alexander P. Veselov for pointing out the link between my solution and moduli spaces of stable curves. Finally, I would like to thank the anonymous referees, who helped to improve the paper considerably with their comments and additional references.

\section{References}

[1] Ballesteros Á., Enciso A., Herranz F.J., Ragnisco O., A maximally superintegrable system on an $n$ dimensional space of nonconstant curvature, Phys. D 237 (2008), 505-509, math-ph/0612080.

[2] Benenti S., Inertia tensors and Stäckel systems in the Euclidean spaces, Rend. Sem. Mat. Univ. Politec. Torino 50 (1992), 315-341.

[3] Benenti S., Orthogonal separable dynamical systems, in Differential Geometry and its Applications (Opava, 1992), Math. Publ., Vol. 1, Silesian Univ. Opava, Opava, 1993, 163-184.

[4] Benenti S., Special symmetric two-tensors, equivalent dynamical systems, cofactor and bi-cofactor systems, Acta Appl. Math. 87 (2005), 33-91.

[5] Benenti S., Chanu C., Rastelli G., Remarks on the connection between the additive separation of the Hamilton-Jacobi equation and the multiplicative separation of the Schrödinger equation. II. First integrals and symmetry operators, J. Math. Phys. 43 (2002), 5223-5253. 
[6] Błaszak M., Bi-Hamiltonian representation of Stäckel systems, Phys. Rev. E 79 (2009), 056607, 9 pages, arXiv:0904.2070.

[7] Bolsinov A.V., Matveev V.S., Geometrical interpretation of Benenti systems, J. Geom. Phys. 44 (2003), 489-506.

[8] Crampin M., Conformal Killing tensors with vanishing torsion and the separation of variables in the Hamilton-Jacobi equation, Differential Geom. Appl. 18 (2003), 87-102.

[9] Crampin M., Projectively equivalent Riemannian spaces as quasi-bi-Hamiltonian systems, Acta Appl. Math. 77 (2003), 237-248.

[10] Crampin M., Sarlet W., Bi-quasi-Hamiltonian systems, J. Math. Phys. 43 (2002), 2505-2517.

[11] Deeley R.J., Horwood J.T., McLenaghan R.G., Smirnov R.G., Theory of algebraic invariants of vector spaces of Killing tensors: methods for computing the fundamental invariants, in Symmetry in Nonlinear Mathematical Physics, Proceedings of Institute of Mathematics, Vol. 50, Editors A.G. Nikitin, V.M. Boyko, R.O. Popovych, I.A. Yehorchenko, Institute of Mathematics, Kyiv, 2004, Part 3, 1079-1086.

[12] Deligne P., Mumford D., The irreducibility of the space of curves of given genus, Inst. Hautes Études Sci. Publ. Math. (1969), 75-109.

[13] Eisenhart L.P., Separable systems of Stäckel, Ann. of Math. 35 (1934), 284-305.

[14] Frolov V.P., Zelnikov A., Introduction to black hole physics, Oxford University Press, Oxford, 2011.

[15] Horwood J.T., McLenaghan R.G., Smirnov R.G., Invariant classification of orthogonally separable Hamiltonian systems in Euclidean space, Comm. Math. Phys. 259 (2005), 670-709, math-ph/0605023.

[16] Ibort A., Magri F., Marmo G., Bihamiltonian structures and Stäckel separability, J. Geom. Phys. 33 (2000), $210-228$.

[17] Kalnins E.G., Separation of variables for Riemannian spaces of constant curvature, Pitman Monographs and Surveys in Pure and Applied Mathematics, Vol. 28, Longman Scientific \& Technical, Harlow, 1986.

[18] Kalnins E.G., Kress J.M., Miller Jr. W., Superintegrability in a non-conformally-flat space, J. Phys. A: Math. Theor. 46 (2013), 022002, 12 pages, arXiv:1211.1452.

[19] Kalnins E.G., Kress J.M., Miller Jr. W., Winternitz P., Superintegrable systems in Darboux spaces, J. Math. Phys. 44 (2003), 5811-5848, math-ph/0307039.

[20] Kalnins E.G., Kress J.M., Winternitz P., Superintegrability in a two-dimensional space of nonconstant curvature, J. Math. Phys. 43 (2002), 970-983, math-ph/0108015.

[21] Kalnins E.G., Kuznetsov V.B., Miller Jr. W., Quadrics on complex Riemannian spaces of constant curvature, separation of variables, and the Gaudin magnet, J. Math. Phys. 35 (1994), 1710-1731, hep-th/9308109.

[22] Kalnins E.G., Miller Jr. W., Separation of variables on $n$-dimensional Riemannian manifolds. I. The $n$-sphere $S^{n}$ and Euclidean $n$-space $\mathbf{R}^{n}$, J. Math. Phys. 27 (1986), 1721-1736.

[23] Kalnins E.G., Miller Jr. W., Winternitz P., The group O(4), separation of variables and the hydrogen atom, SIAM J. Appl. Math. 30 (1976), 630-664.

[24] Knudsen F.F., The projectivity of the moduli space of stable curves. II. The stacks $M_{g, n}$, Math. Scand. 52 (1983), 161-199.

[25] Knudsen F.F., The projectivity of the moduli space of stable curves. III. The line bundles on $M_{g, n}$, and a proof of the projectivity of $\bar{M}_{g, n}$ in characteristic 0, Math. Scand. 52 (1983), 200-212.

[26] Koenigs G.X.P., Sur les géodésiques a integrales quadratiques, in Le cons sur la théorie générale des surfaces, Vol. 4, Editor J.G. Darboux, Chelsea Publishing, 1972, 368-404.

[27] Kuznetsov V.B., Equivalence of two graphical calculi, J. Phys. A: Math. Gen. 25 (1992), 6005-6026.

[28] Kuznetsov V.B., Quadrics on real Riemannian spaces of constant curvature: separation of variables and connection with Gaudin magnet, J. Math. Phys. 33 (1992), 3240-3254.

[29] Lamé G., Sur les surfaces isothermes dans les corps homogènes en équilibre de température, J. Math. Pures Appl. 2 (1837), 147-188.

[30] Levi-Civita T., Sulla integrazione della equazione di Hamilton-Jacobi per separazione di variabili, Math. Ann. 59 (1904), 383-397.

[31] Lundmark H., Newton systems of cofactor type in Euclidean and Riemannian spaces, Dissertations, Vol. 719, Linköping Studies in Science and Technology, Linköping, 2001.

[32] Matveev V.S., Mounoud P., Gallot-Tanno theorem for closed incomplete pseudo-Riemannian manifolds and applications, Ann. Global Anal. Geom. 38 (2010), 259-271, arXiv:0909.5344. 
[33] Matveev V.S., Topalov P.І̆., Trajectory equivalence and corresponding integrals, Regul. Chaotic Dyn. 3 (1998), 30-45.

[34] McLenaghan R.G., Milson R., Smirnov R.G., Killing tensors as irreducible representations of the general linear group, C. R. Math. Acad. Sci. Paris 339 (2004), 621-624.

[35] Milson R., Schöbel K., Integrable Killing tensors on 3-dimensional constant curvature spaces, in preparation.

[36] Neumann C., De problemate quodam mechanico, quod ad primam integralium ultraellipticorum classem revocatur, J. Reine Angew. Math. 56 (1859), 46-63.

[37] Nijenhuis A., $X_{n-1}$-forming sets of eigenvectors, Nederl. Akad. Wetensch. Proc. Ser. A. 54 (1951), 200-212.

[38] Olevskiŭ M.N., Triorthogonal systems in spaces of constant curvature in which the equation $\Delta_{2} u+\lambda u=0$ allows a complete separation of variables, Mat. Sb. 27 (1950), 379-426.

[39] Painlevé P., Sur les intégrales quadratiques des équations de la dynamique, Compt. Rend. 124 (1897), 221-224.

[40] Rauch-Wojciechowski S., Marciniak K., Lundmark H., Quasi-Lagrangian systems of Newton equations, J. Math. Phys. 40 (1999), 6366-6398, solv-int/9909025.

[41] Schöbel K.P., Algebraic integrability conditions for Killing tensors on constant sectional curvature manifolds, J. Geom. Phys. 62 (2012), 1013-1037, arXiv:1004.2872.

[42] Schöbel K.P., Veselov A.P., Separation coordinates, moduli spaces and Stasheff polytopes, arXiv:1307.6132.

[43] Singer I.M., Thorpe J.A., The curvature of 4-dimensional Einstein spaces, in Global Analysis (Papers in Honor of K. Kodaira), Univ. Tokyo Press, Tokyo, 1969, 355-365.

[44] Sinjukov N.S., On the theory of a geodesic mapping of Riemannian spaces, Dokl. Akad. Nauk SSSR 169 (1966), 770-772.

[45] Stäckel P., Die Integration der Hamilton-Jacobischen Differentialgleichung mittelst Separation der Variablen, habilitationsschrift, Universität Halle, Halle, 1891.

[46] Stasheff J.D., Homotopy associativity of $H$-spaces. I, Trans. Amer. Math. Soc. 108 (1963), 275-292. 